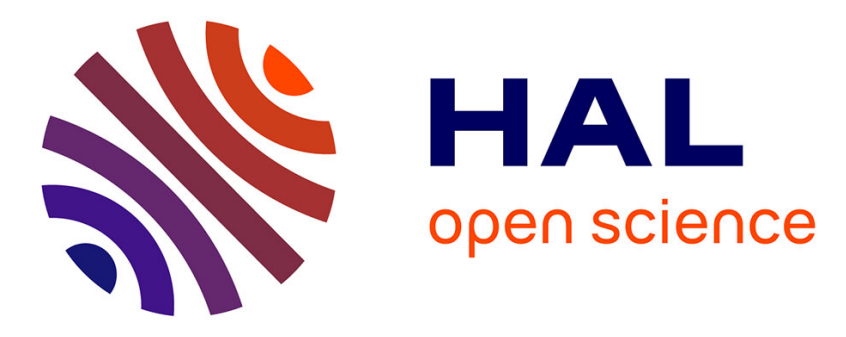

\title{
Probing the interactions between air bubbles and (bio)interfaces at the nanoscale using FluidFM technology
}

Irem Demir, Ines Lüchtefeld, Claude Lemen, Etienne Dague, Pascal Guiraud, Tomaso Zambelli, Cécile Formosa-Dague

\section{To cite this version:}

Irem Demir, Ines Lüchtefeld, Claude Lemen, Etienne Dague, Pascal Guiraud, et al.. Probing the interactions between air bubbles and (bio)interfaces at the nanoscale using FluidFM technology. Journal of Colloid and Interface Science, 2021, 604, pp.785-797. 10.1016/j.jcis.2021.07.036 . hal-03423284

\section{HAL Id: hal-03423284 \\ https://hal.science/hal-03423284}

Submitted on 15 Nov 2021

HAL is a multi-disciplinary open access archive for the deposit and dissemination of scientific research documents, whether they are published or not. The documents may come from teaching and research institutions in France or abroad, or from public or private research centers.
L'archive ouverte pluridisciplinaire HAL, est destinée au dépôt et à la diffusion de documents scientifiques de niveau recherche, publiés ou non, émanant des établissements d'enseignement et de recherche français ou étrangers, des laboratoires publics ou privés. 


\title{
Probing the interactions between air bubbles and (bio)interfaces at the nanoscale using FluidFM technology
}

\author{
Irem Demir, ${ }^{1,2}$ Ines Lüchtefeld, ${ }^{3}$ Claude Lemen, ${ }^{1}$ Etienne Dague, ${ }^{2,4}$ Pascal Guiraud, ${ }^{1,4}$ \\ Tomaso Zambelli, ${ }^{3}$ and Cécile Formosa-Dague ${ }^{1,4 *}$
}

${ }^{1}$ TBI, Université de Toulouse, INSA, INRAE, CNRS, Toulouse, France.

${ }^{2}$ LAAS, Université de Toulouse, CNRS, Toulouse, France.

${ }^{3}$ Laboratory of Biosensors and Bioelectronics, Institute for Biomedical Engineering, ETH Zurich, Zurich, Switzerland.

${ }^{4}$ Fédération de Recherche Fermat, CNRS, Toulouse, France.

*corresponding author: Cécile Formosa-Dague, formosa@insa-toulouse.fr

Postal address: Toulouse Biotechnology Institute, INSA de Toulouse, 135 avenue de Rangueil, 31077 Toulouse Cedex 4, France

Phone: +33561559967 


\begin{abstract}
Understanding the molecular mechanisms underlying bubble-(bio)surfaces interactions is currently a challenge that if overcame, would allow to understand and control the various processes in which they are involved. Atomic force microscopy is a useful technique to measure such interactions, but it is limited by the large size and instability of the bubbles that it can use, attached either on cantilevers or on surfaces. We here present new developments where microsized and stable bubbles are produced using FluidFM technology, which combines AFM and microfluidics. The air bubbles produced were used to probe the interactions with hydrophobic samples, showing that bubbles in water behave like hydrophobic surfaces. They thus could be used to measure the hydrophobic properties of microorganisms' surfaces, but in this case the interactions are also influenced by electrostatic forces. Finally a strategy was developed to functionalize their surface, thereby modulating their interactions with microorganism interfaces. This new method provides a valuable tool to understand bubble-(bio)surfaces interactions but also to engineer them.
\end{abstract}

\title{
KEYWORDS
}

Air bubbles, Interactions, Fluidic force microscopy, Atomic force microscopy, Hydrophobicity, Microorganisms 


\section{INTRODUCTION}

Over the past decades, air bubbles have attracted much attention because of their remarkable characteristics. In liquid, air bubbles feature $i$ ) a great stability [1], ii) an affinity for hydrophobic surfaces [2], iii) a high interface area per unit of volume [3], iv) an ability to interact with organic molecules (proteins, lipids, and polymers), or surfactants [4], and v) a capacity to adhere to solid surfaces [5]. Because of these properties, bubbles play crucial roles in several application fields such as agriculture [6], microdynamics of fluids [7], material science [8] or (bio)chemical engineering [9]. For example in chemical engineering, bubbles can be used to separate cells from their liquid culture medium [10], or when combined with membrane filtration, they can prevent or reduce membrane fouling [11]. Bubbles have also been used in health-related clinical applications in recent years, where they act as efficient carriers for genes and drugs [1214]. A common point in all these applications is that bubbles interact either with an abiotic surface or with living cells. Thus understanding the physico-chemical basis of these bubble-(bio)surfaces interactions becomes an important aspect, as this comprehension would allow to understand and control the processes they are involved in.

While fluid dynamics aspects of bubbles-(bio)surfaces interactions are to some extent understood $[15,16]$, the underlying molecular mechanisms have not been fully deciphered. One possibility to access these interactions is to use atomic force microscopy (AFM) [17]. AFM, first developed in 1986 [18], has demonstrated over the years to be a powerful tool for surface characterization and force detection at the nanoscale. It was first used to measure the interactions between air bubbles and particle(s)/surface(s) in 1994 [19]. For that the authors attached silica particles on cantilevers [20] and used these colloidal probes to measure the interactions with bubbles of several hundreds of $\mu \mathrm{m}$ in diameter, stabilized on hydrophobic surfaces [19]. By analyzing the force curves obtained with hydrophilic and hydrophobic silica spheres, they could determine that gas bubbles in water behave like hydrophobic surfaces. To access the interactions between bubbles and different surface samples, Vakarelski et al. developed in 2008 another strategy where single bubbles of $90-120 \mu \mathrm{m}$ in diameter, are picked up on hydrophobized V-shaped cantilevers [21]; this method was used to probe the interactions with other bubbles and provided details on how bubbles interact in natural conditions. This strategy has also been used to probe the interactions between bubbles and microorganisms [22,23]. In a first study by Ditscherlein et al., the interactions between air bubbles of $20-150 \mu \mathrm{m}$ in diameter and layers of yeast cells were probed [22]; the results obtained indicated that these interactions are hydrophobic, but can be affected by $\mathrm{pH}$, salt concentration or ionic strength. Later on, Yumiyama et al., using the same technique, measured the interactions between air bubbles of $50 \mu \mathrm{m}$ in diameter, and single yeast cells instead of layers [23]. Their results also showed that hydrophobic interactions were involved; however, in this case, bubbles were ten times bigger than yeast cells, thus depending on the force applied, bubbles could also interact with the surface on which cells were immobilized.

In all these examples, even though experimental parameters such as $\mathrm{pH}$ or ionic strength, as well as system parameters such as applied force, approach velocity or contact time are adjustable, issues related to the bubble size remain, as it cannot be controlled over time because of the Laplace pressure [24,25]. Laplace pressure is the differential pressure across inside and outside of a curved interface that forms a boundary between gas and liquid regions [26]. Over time the gases inside the bubble dissolve in the water which modifies their size, and thus the contact area between the bubble and the sample. In the case of interactions with cells, the change in the contact area could modify the number of molecules from the cell surface involved in the interaction, which 
can have an impact on the final adhesion force recorded [27,28]. This aspect is also related to a second issue that is the variability in size of the bubbles used to probe the interactions. Indeed, using these methods, the size of a bubble is not controllable from one bubble to another, thus for each bubble the contact area is different, thereby possibly leading to differences in the adhesion forces. Finally, in the case where interactions with single microorganisms are probed, the size of the bubbles is too large compared to the size of cells, which can also lead to the introduction of a bias in the results.

To overcome these challenges, we here develop a new method to probe the interactions between bubbles and (bio)interfaces using fluidic force microscopy technology (FluidFM) that combines AFM with microfluidics. In this system, a microsized channel is integrated into an AFM cantilever and connected to a pressure controller, thus creating a continuous and closed fluidic conduit that can be filled with a solution, while the tool can be immersed in a liquid environment [29]. An aperture at the end of the cantilever allows liquids to be dispensed locally. In this configuration, FluidFM technology has been used in various types of studies in material science, for example to functionalize surfaces with polymer layers [30], or in life sciences for example to perform single-cell force spectroscopy experiments [31,32]. In this study, we use FluidFM in an original manner, and develop a method to produce stable microsized bubbles at the aperture of FluidFM cantilevers. We first describe this method; after the characterization of the bubbles produced, we probed their interactions with hydrophobic surfaces and living cells. Finally, we also demonstrate the possibility to functionalize the surface of these bubbles in order to modify their interactions with microorganisms.

\section{MATERIAL and METHODS}

Microalgae strain and culture. The green freshwater microalgae Chlorella vulgaris strain CCAP 211/11B (Culture Collection of Algae and Protozoa) was cultivated in Wright's cryptophyte (WC) medium prepared with deionized water [33], at $20^{\circ} \mathrm{C}$, under $120 \mathrm{rpm}$ agitation, in an incubator equipped with white neon light tubes providing illumination of approximately $40 \mu$ mol photons $\mathrm{m}^{-2} \mathrm{~s}^{-1}$ with a photoperiod of $18 \mathrm{~h}$ light: $6 \mathrm{~h}$ dark. All experiments were carried out with 7 days exponential phase batch cultures. Before experiments, cells were first harvested by centrifugation (3000 rpm, $3 \mathrm{~min}$ ), washed two times in PBS at pH 7.8, and immobilized on polyethylenimine (PEI, Sigma-Aldrich P3143) coated glass slides prepared as previously described [34]. Briefly, freshly oxygen activated glass slides were covered by a $0.2 \%$ PEI solution in deionized water and left for incubation overnight. Then the glass slides were rinsed with deionized water and dried under nitrogen. A total of $1 \mathrm{~mL}$ of the cell suspension was then deposited on the PEI slides, allowed to stand for at least $30 \mathrm{~min}$ at room temperature, and rinsed with PBS.

Yeast strain and culture. Candida albicans (from ABC Platform ${ }^{\circledR}$ Bugs Bank, Nancy, France) was stocked at $-80^{\circ} \mathrm{C}$, revivified on a standard rich YPD (Yeast extract Peptone Dextrose) agar (Difco; 242720) and grown in YPD broth (Difco; 242820) for $20 \mathrm{~h}$ at $30^{\circ} \mathrm{C}$ under $230 \mathrm{rpm}$ agitation. Yeast cells were then harvested by centrifugation (4500 rpm, $3 \mathrm{~min}$ ), washed two times in acetate buffer (18 $\left.\mathrm{mM} \mathrm{CH} \mathrm{COONa}_{3} 1 \mathrm{mM} \mathrm{CaCl}_{2}, 1 \mathrm{mM} \mathrm{MnCl}_{2}, \mathrm{pH} 5.2\right)$, and immobilized on Concanavalin A (ConA) coated surfaces. For that, $200 \mu \mathrm{L}$ of ConA $(10 \mu \mathrm{g} / \mathrm{mL})$ were deposited on polystyrene Tissue Culture dish (Sterilized by radiation, TPP Switzerland) and incubated overnight. Then the Petri dishes were rinsed with deionized water and dried under nitrogen. A total of $1 \mathrm{~mL}$ of the cell suspension was then deposited on the ConA coated Petri dishes, allowed to stand for at least $30 \mathrm{~min}$ at room temperature, and rinsed with acetate buffer. 
Bacterial strain and culture. Pseudomonas aeruginosa cells (ATCC 27853) were stocked at $-80^{\circ} \mathrm{C}$, revivified on Mueller Hinton Agar (Difco, 225250) and grown in Mueller Hinton Broth (Difco, 275730) for 18 hours (stationary phase) at $35^{\circ} \mathrm{C}$ under static conditions. Cells were harvested by centrifugation ( $3000 \mathrm{rpm}, 3 \mathrm{~min}$ ) and washed two times in PBS at pH 7.8. Before experiments cells were immobilized on PEI coated glass slides.

Preparation of hydrophobic surfaces. Monolayers of CH3- (1-dodecanethiol, Sigma-Aldrich, 471364) and OH-terminated (11-mercapto-1-undecanol, Sigma-Aldrich, 447528) alkanethiols, mixed in different proportions were self-assembled on gold surfaces to obtain different contact angles, as described in Dague et al. 2007 . For that, silicon wafers (Siltronix, France) were first coated by electron beam thermal evaporation with a 5 -nm-thick Cr layer followed by a 30-nmthick $\mathrm{Au}$ layer. These gold-coated surfaces were then cleaned by oxygen plasma ( $3 \mathrm{~min})$, rinsed with ethanol and dried under nitrogen. They were finally immersed for $14 \mathrm{~h}$ in ethanol solutions containing $1 \mathrm{mM}$ 1-dodecanethiol $\left(\mathrm{CH}_{3^{-}}\right)$and 11-mercapto-1-undecanol $(\mathrm{OH}-)$ in different proportions depending on the degree of hydrophobicity wanted, and rinsed with ethanol before use.

Water-contact angle measurement. Water contact angles were measured at a $0.1^{\circ}$ resolution with a white light source (Digidrop GBX, France). Sesile drop method has been used to measure the water contact angle. Shortly, a water droplet (approx. $4 \mu 1$ volume) is first released on top of the sample. Then, an edge-detection method is applied to find the drop contour in the image and thus measure the contact angle. For each surface three measurements were performed prior to each experiment.

Bubble formation using FluidFM. First, tipless FluidFM probes with an aperture of $8 \mu \mathrm{m}$ of diameter (Cytosurge AG, Switzerland) were made hydrophobic by coating them with selfassembled monolayers (SAMs) of silanes via SAMs vapor deposition technique. FluidFM cantilevers were functionalized with $1 \mathrm{H}, 1 \mathrm{H}, 2 \mathrm{H}, 2 \mathrm{H}$-Perfluorodecyltrichlorosilane (FDTS) using an Orbis-1000 equipment (Memsstar, Livingston, UK) to make their external surface and inside microchannel hydrophobic. The deposition was realized under vacuum at 40 torrs and $-40^{\circ} \mathrm{C}$, for 5 min [36]. Then the microchannel of these silanized cantilevers was filled with air and the probe was immersed in a liquid environment (buffer used to perform the experiments). To eliminate any particle or dust contamination or to prevent clogging of the FluidFM cantilever, a slight over pressure of $20 \mathrm{mbar}$ is applied. Then to produce a bubble at the aperture of the cantilever, a positive pressure from 100 to 200 mbar was applied inside the microfluidic cantilever in buffer. The silanized probes were calibrated using the thermal noise method [37] before each measurement.

Side images. Side images of the microbubbles were recorded using a $0.50 \mathrm{~mm}$ right angle reflective prism (Al coated hypotenuse, Edmund Optics, USA). Prism is illuminated with manual lateral light source. This reflective prism and side light source made it possible to image the cantilever and bubble from the side, and measure its perpendicular size depending on the pressure applied. Fig. 2a shows a scheme of the experimental procedure used to obtain these side images.

Force spectroscopy experiments. Force spectroscopy experiments were conducted using a NanoWizard III AFM (Bruker, USA), equipped with FluidFM technology (Cytosurge AG, Switzerland). In each case, experiments were performed in either Phosphate Buffer Saline (PBS) or acetate buffer, using micropipette probes with an aperture of $8 \mu \mathrm{m}$ (spring constant of $0.3,2$, and $4 \mathrm{~N} / \mathrm{m}$ ) (Cytosurge AG, Switzerland). Interactions between the formed bubbles and hydrophobic surfaces were recorded with probes with a spring constant of 2 and $4 \mathrm{~N} / \mathrm{m}$ at a 
constant applied force of $1 \mathrm{nN}$, while interactions with cells were measured using probes with a spring constant of 0.3 and $2 \mathrm{~N} / \mathrm{m}$, at a constant applied force of $1 \mathrm{nN}$. Force curves were recorded with a retraction z-length of up to $20 \mu \mathrm{m}$ and a constant retraction speed of $4.0 \mu \mathrm{m} / \mathrm{s}$ to $20 \mu \mathrm{m} / \mathrm{s}$. High z-lengths of $20 \mu \mathrm{m}$ were reached using the CellHesion module on the NanoWizard III AFM (Bruker). CellHesion Module has a vertical range of movement of $100 \mu \mathrm{m}$ due to the piezo-driven movement of the sample holder. Data were analyzed using the Data Processing software from Bruker. Adhesion forces were obtained by calculating the maximum adhesion force for each curve. For the interactions with surfaces, experiments were repeated at least five times with one surface in each case. For the interactions with microorganisms, experiments were repeated three times with ten different cells coming from at least three different cultures.

AFM imaging. AFM images were recorded on cells immobilized on PEI-coated glass slides (for $P$. aeruginosa and C. vulgaris cells) or on ConA coated petri dishes (for C. albicans). For $C$. albicans and C. vulgaris, images were recorded in acetate buffer at $\mathrm{pH} 5.2$ and in $\mathrm{PBS}$ at $\mathrm{pH} 7.4$ respectively, using the Quantitative Imaging mode available on the Nanowizard III AFM (Bruker), with MSCT cantilevers (Bruker, nominal spring constant of $0.01 \mathrm{~N} / \mathrm{m}$ ). Images were recorded at 128 pixels $\times 128$ pixels with an applied force kept at $1.5 \mathrm{nN}$ for all conditions and a constant approach/retract speed of $90 \mu \mathrm{m} / \mathrm{s}$ (z-range of $3 \mu \mathrm{m}$ ). For $P$. aeruginosa, images were recorded in contact mode, using a set point inferior to $1 \mathrm{nN}$. In all cases the cantilevers spring constants were determined by the thermal noise method prior to imaging [37].

AFM cantilever functionalization. Tips functionalized with phosphorus dendrimers, so-called "dendritips", were prepared as previously described [38]. Briefly, MLCT AUWH cantilevers (Bruker, USA, spring constant of $0.01 \mathrm{~N} / \mathrm{m}$ ) were first cleaned using oxygen plasma ( $3 \mathrm{~min}, 0.5$ mbar), placed in a $5.52 \mathrm{M}$ ethanolamine solution $(3.3 \mathrm{~mL}$ of ethanolamine hydrochloride were dissolved in $6.6 \mathrm{~mL}$ Dimethyl sulfoxide(DMSO)) and incubated overnight at room temperature. The tips were then washed with DMSO and ethanol, and dried under nitrogen. These amino tips were next incubated for 5-6 h in the dendrimer solution (58 $\mu \mathrm{M}$ in Tetrahydrofuran (THF)), rinsed in THF and ethanol, and dried under nitrogen. Such "dendritips" were finally functionalized with colistin. For that, they were incubated in a drop of $100 \mu \mathrm{L}$ of colistin sulfate salt $(0.1 \mathrm{mg} / \mathrm{L}$, Sigma Aldrich, C4461) for $1 \mathrm{~h}$ at room temperature. Then $10 \mu \mathrm{L}$ of $\mathrm{NaCNBH}_{3}(20 \mathrm{mM}$ final concentration) were added in the colistin drop for $15 \mathrm{~min}$ to let the reduction take place. Finally, tips were washed in PBS and used to probe the interactions with cells.

Bubble functionalization. A zwitterionic head group labeled with 1,2-dipalmitoyl-sn-glycero-3phosphoethanolamine- $\mathrm{N}$ - (lissamine rhodamine B sulfonyl) (ammonium salt) was used as a fluorescent surfactant. First, the FluidFM cantilever was immersed in a solution of $0.01 \mathrm{~g} / \mathrm{L}$ of the fluorescent surfactant. This solution was aspirated inside the cantilever by gradually decreasing the pressure from 0 mbar to $-200 \mathrm{mbar}$. After that the FluidFM cantilever containing the surfactant solution was immersed in PBS buffer without surfactants. By increasing the pressure to $200 \mathrm{mbar}$, the surfactant solution was then locally dispersed in the buffer and a bubble was formed: the surfactant then assembled at the surface of the produced bubble. To functionalize the bubble surface with colistin, a solution of $20 \mathrm{mg} / \mathrm{L}$ of colistin sulfate salt $(\geq 19,000 \mathrm{IU} / \mathrm{mg}$, Sigma-Aldrich) was used; the same procedure was applied.

Side image analysis. Images were first calibrated to calculate the size of one pixel. The optical focus was kept constant throughout the calibration procedure and the cantilever was retracted with a $10 \mu \mathrm{m}$-step increment. This allowed to calculate the pixel scale size. Sub ROI extraction method 
was then used to calculate the bubble sizes with color channel separation of red (R) green $(\mathrm{G})$ and blue (B).

\section{RESULTS and DISCUSSION}

\section{FluidFM allows to produce stable microbubbles at the aperture of microfluidic cantilevers}

To produce microsized bubbles at the aperture of FluidFM cantilevers (Fig. 1A), we first functionalized FluidFM cantilevers with self-assembled monolayers (SAMSs) of silanes (FDTS) to make them hydrophobic. Then, as depicted in Figure 1A, the microfluidic channel inside the cantilever is filled with air, and immersed in liquid. By applying a positive pressure (200 mbar) inside the cantilever, bubbles were formed directly at the aperture. Because the pressure is maintained constant in the cantilever during experiments, the gas dissolution from the bubble is compensated, which allows keeping its size constant over time. Moreover, the hydrophobic coating allows maintaining the bubble produced at the aperture of the cantilever, otherwise it could rise up in the suspension. In our case, buffers were used (PBS or acetate buffers depending later on the type of cells probed) as we observed bubbles in pure water collapse upon touching the surface. Tipless cantilevers with a circular aperture $8 \mu \mathrm{m}$ of diameter were used; with smaller apertures, the Laplace pressure was so important that the pressure needed to form the bubble was beyond the maximum value achievable with our system (800 mbar). Figure $1 \mathrm{~B}$ and $\mathrm{C}$ shows optical images of the microfluidic cantilever from a bottom view (Fig. 1B) and from a side-view (Fig. 1C). While the bubble on the bottom view is not clearly visible, the side image of the cantilever shows the presence of the bubble at the aperture of the cantilever so that we could follow the bubble formation in situ, and afterwards fully characterize its shape and size.
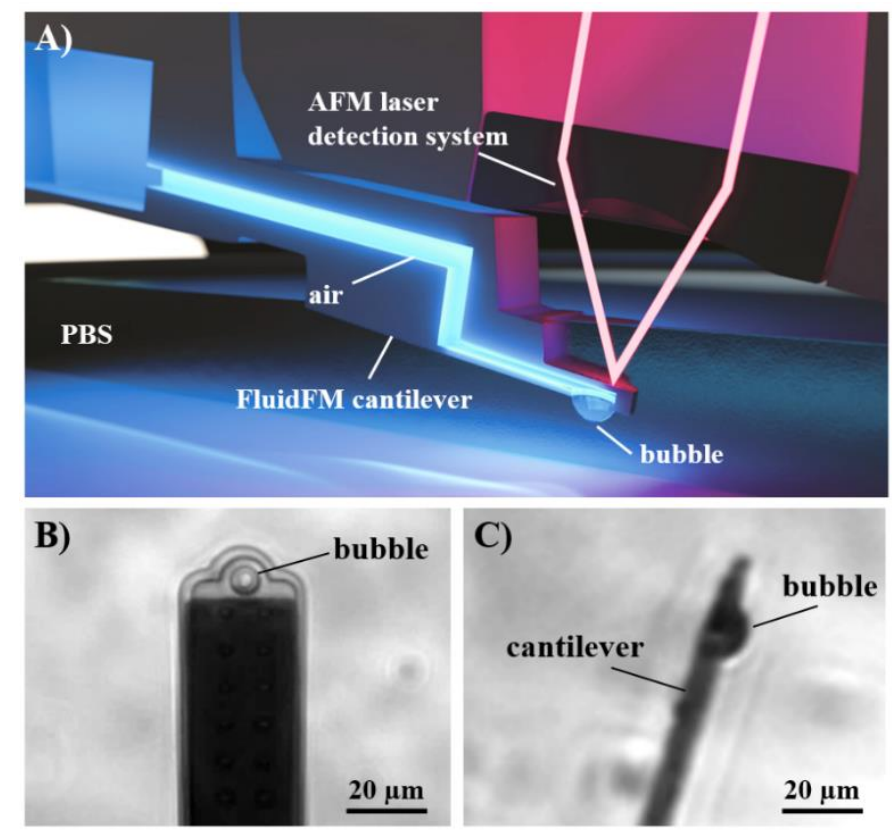

Figure 1. Schematic representation of the method to produce bubbles using FluidFM technology. A) A FluidFM microchanneled cantilever with a circular aperture of $8 \mu \mathrm{m}$ diameter, connected to a pressure controller, is filled with air and immersed in liquid. By applying a positive pressure, an air bubble can be formed at the aperture of the cantilever. B) Bottom image of a FluidFM probe with a bubble formed at its aperture (200 mbar) and C) corresponding side image. 
To obtain such side images, we used a $45^{\circ}$ prism with a reflective side $(0.5 \mathrm{~mm})$ manually positioned next to the FluidFM probe and laterally illuminated with an external light source. This set-up is described in Figure 2A; it is inspired by recent approaches described in the literature where side-view imaging paths were developed for AFM integration[39,40]. The side-view images presented in Figure 2B-E show bubble formation depending on the applied pressure in the cantilever. Since these images are not direct images, but images upon reflection from the prism, there are several factors affecting the size observed on the images, i.e, the position of the FluidFM cantilever, the position of the reflective prism, the angle between the reflective prism and the cantilever (if it is fully perpendicular to the cantilever or not) and finally the angle of the side light source. Thus, the first step is to calibrate the images obtained to accurately determine the size of one pixel. For that, the FluidFM cantilever was moved in the Z-direction with known distances (from position $\mathrm{x}=0 \mu \mathrm{m}$ to $\mathrm{x}=70 \mu \mathrm{m}$ ) using an increment of $10 \mu \mathrm{m}$. By measuring the distance on the images it was possible to determine the size of each pixel, and thus to measure the dimensions of the bubbles produced (see Supplementary Fig. S1). Knowing the pixel size on the side-images obtained, we thus measured the sizes of bubbles as a function of the pressure $\mathrm{p}, 100$ $<\mathrm{p}<200$ mbar. For $\mathrm{p}<100$ mbar, no bubbles were observed (Fig. 2B). On the contrary, for $\mathrm{p}>$ 100 mbar, a bubble appears at the aperture of the probe: optically we inferred a radius $(r)$ of $4 \mu \mathrm{m}$ and a perpendicular size $(H)$ of $1.2 \mu \mathrm{m}$ (Fig. 2C). When the pressure is further increased, $r$ remains constant $(4 \mu \mathrm{m})$, matching the aperture size, whereas $\mathrm{H}$ increases from 2.2 to $3.2 \mu \mathrm{m}$. At $200 \mathrm{mbar}$, the bubbles produced are stable, meaning that their size is constant over time, as showed by time course side images recorded during 1 hour (Supplementary Fig. S2). Finally, above a pressure of 200 mbar, bubbles are not stable anymore, i.e. they detach from the cantilever and rise up in the suspension. When we consider the Laplace law, which gives the Laplace pressure as a ratio between the surface tension and the interface radius, as the pressure increases, the bubble radius should decrease. What we see is that when the pressure increases, the bubble radius $r$ stays constant while its perpendicular size $H$ increases. To understand what is happening when a bubble is formed, we need to consider that the bubble produced is actually a spherical cap as described in Figure $2 \mathrm{~F}$, where only the part depicted in yellow is visible at the aperture of the cantilever. When a low pressure is applied, the perpendicular size $H$ of the produced spherical cap is low, but in fact its surface radius, $R$, in this case is large (for a flat interface, the radius is infinite). As we increase the pressure inside the cantilever, the perpendicular size $H$ increases, in turn meaning that the surface radius $R$ of the spherical cap decreases. Thus indeed as the pressure increases, its surface radius decreases. With this in mind, it is then possible to calculate the critical pressure to apply to keep the bubble stable, using the Laplace law given by the following equation:

$$
\Delta P=\frac{2 \gamma}{R}(1)
$$

Where $\Delta P$ is the difference of pressure inside and outside the bubble, $\gamma$ is the surface tension of the surrounding liquid, and $R$ the surface radius of the spherical cap. We can then apply the Pythagoras theorem to determine the length of its radius:

$$
\begin{gathered}
R^{2}=r^{2}+\left(R^{2}-H^{2}\right)(2) \\
R=\frac{r^{2}+H^{2}}{2 H}(3)
\end{gathered}
$$

Where $R$ is the spherical cap surface radius which corresponds to the hypotenuse, $H$ is the perpendicular size of the spherical cap, which corresponds to its height, and $r$ is the bubble radius at the cantilever aperture (Figure 2F). Both $r$ and $H$ are measured on the side images. Equation 3 
can be used during the first step of the inflation process where $H<r$ and $R>r$, until $H=r$ where the Laplace pressure is maximum because the surface radius $R$ of the spherical cap is minimal. During this first inflation step, the combination of equation 1 and 3 results in the following formula for the Laplace pressure:

$$
\Delta P=\frac{4 \gamma H}{r^{2}+H^{2}}(4)
$$

This means that the applied pressure needed to increase the bubble size, also needs to increase to overcome the Laplace pressure, corresponding to what was observed in the experiments. It shows that the maximum spherical cap height $H$ that can be reached is of $4 \mu \mathrm{m}$, which corresponds to the value of the spherical cap radius $r$ on the cantilever, and thus to the radius of its aperture, for a theoretical applied pressure of 340 mbar. If the applied pressure keeps increasing, the spherical cap surface radius $R$ increases again so that the internal Laplace pressure decreases. The applied pressure suddenly becomes larger than the internal pressure, leading to a rapid increase of the gas flow towards the bubble, which inflates in an unstable process as the Laplace internal pressure continues to decrease. The consequence is that the bubble rapidly becomes too large to stay attached to the cantilever and rises up in the solution due to the Archimedes force. This fully explains the experimental behaviors observed, even if the observed maximum pressure that can be applied is closer to 250 mbar.
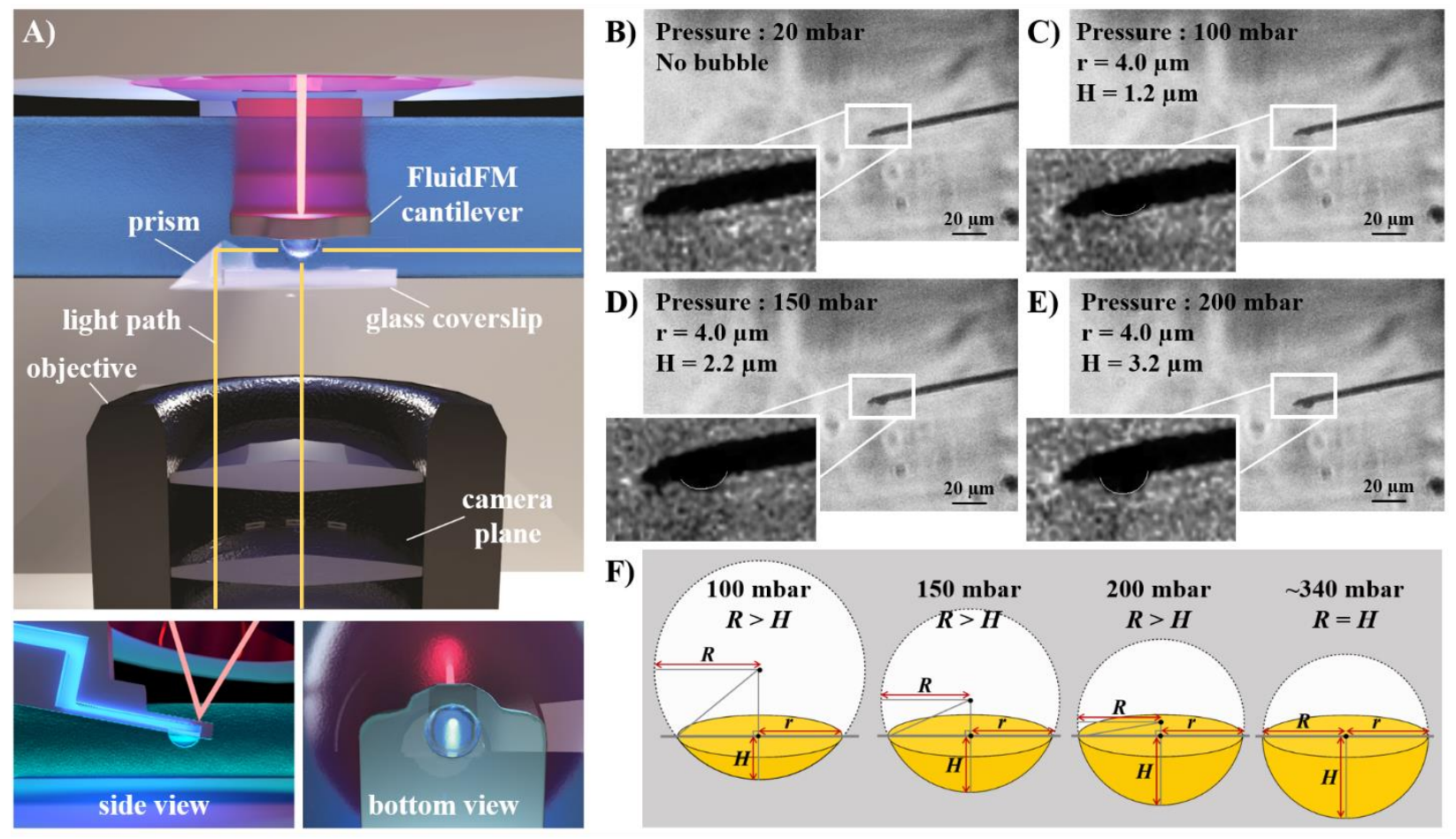

Figure 2. Characterization of the bubbles produced at the FluidFM cantilever aperture. A) Schematic representation of the experimental set-up used to obtain side images of the cantilever. A reflective prism is placed next to the FluidFM probe; by illuminating it from the side, it is possible to visualize the cantilever from the side. Side images of the FluidFM cantilever at B) 20 mbar, C) 100 mbar, D) 150 mbar and E) 200 mbar. In each case, the spherical cap radius $r$ and perpendicular size $H$ are measured. F) Schematic representation of the spherical cap bubble depending on the pressure applied. 
The method we propose here allows to produce small bubbles, which have a constant size over time. This is possible because the FluidFM system (controller, tubing and FluidFM chip) in our operating conditions contains a large volume of air (of several $\mathrm{mL}$ ) at a defined pressure. Given the small size of the bubbles produced, it means that the volume of gas dissolving from the bubble is negligible compared to this overall gas volume, which thus ensures that the bubble diameter stays constant. In addition, the microfluidic controller of the FluidFM regulates the applied pressure inside the system; this regulation system this way refills any lost air. Thanks to this steady supply of air, the diameter of the bubble produced is determined by the active pressure and surface tension, and is not limited by the available volume of air, even if in our case, it should be large enough to maintain the bubble constant over a very long period of time. Another important aspect is that the bubbles produced are small, thus their interface is rigid, which means that they should not deform during force spectroscopy experiments. Bubble deformation has already been the subject of several studies in the literature [22,27,41]. In a recent study, Ditscherlein et al. calculated the bubble deformation using the Johnson-Kendall-Roberts (JKR) theory. Their findings showed that the deformation of the bubble depends on the applied force and for low applied forces (below $50 \mathrm{nN}$ ), the deformation fits well with the theoretical model especially for bubbles with smaller radii (below $60 \mu \mathrm{m}$ ) [22]. In our case, the bubbles produced have a maximum radius $R$ of $4 \mu \mathrm{m}$. Using the Laplace equation, we can calculate the internal pressure of these bubbles which is over 400 mbar, meaning that to deform the bubble, the pressure applied on it needs to be more important than this value. We can convert this pressure into a force applied, as the force corresponds to the pressure multiplied by the surface area. Considering a sphere of $4 \mu \mathrm{m}$ of radius, we find that the force applied needed to deform the bubble would be of $2000 \mathrm{nN}$. Because the bubbles produced by FluidFM are small, stable and non-deformable, in force spectroscopy studies the contact area between the bubble and the sample surface will be the same at a given applied force, thereby allowing to accurately measure interaction forces.

\section{Bubbles produced behave like hydrophobic surfaces.}

Once the protocol for the formation of stable bubble validated, we considered force spectroscopy experiments to investigate the interactions between bubbles and surfaces (Fig. 3A). We focused on hydrophobic surfaces because, when measuring the interactions between bubbles and hydrophobic or hydrophilic silica particles, Ducker and co-authors concluded that an air bubble in water is likely to behave like a hydrophobic surface [19]. To verify this finding, we first produced surfaces with different water contact angles (WCA) by functionalizing flat gold coated surfaces with $\mathrm{CH}_{3}$ - or $\mathrm{OH}$ - terminated alkanethiols, as described in [35,42]. By mixing the two different thiols in different proportions, it was possible to obtain hydrophobic surfaces with a WCA ranging from 42.5 to $79.8^{\circ}$, as measured using the sessile drop method. The retract force curves obtained on the different surfaces are shown in Figure 3B. The peaks observed are typical of nonspecific interactions such as hydrophobic interactions, and the adhesion force recorded increases with the WCA of the surface. The quantification of these adhesion forces is presented in Figure 3C (adhesion forces values obtained for each surfaces can be found in Supplementary Table S1), where it is clear that increasing the WCA of the surfaces results in an increase in the adhesion forces, from $36.4 \pm 9.0 \mathrm{nN}$ for a surface with a WCA of $42.5^{\circ}$, to $268.3 \pm 31.9 \mathrm{nN}$ for a surface with a WCA of $79.8^{\circ}$. We can also see on this histogram that small differences in the WCAs measured resulted in important differences in the adhesion forces recorded. 

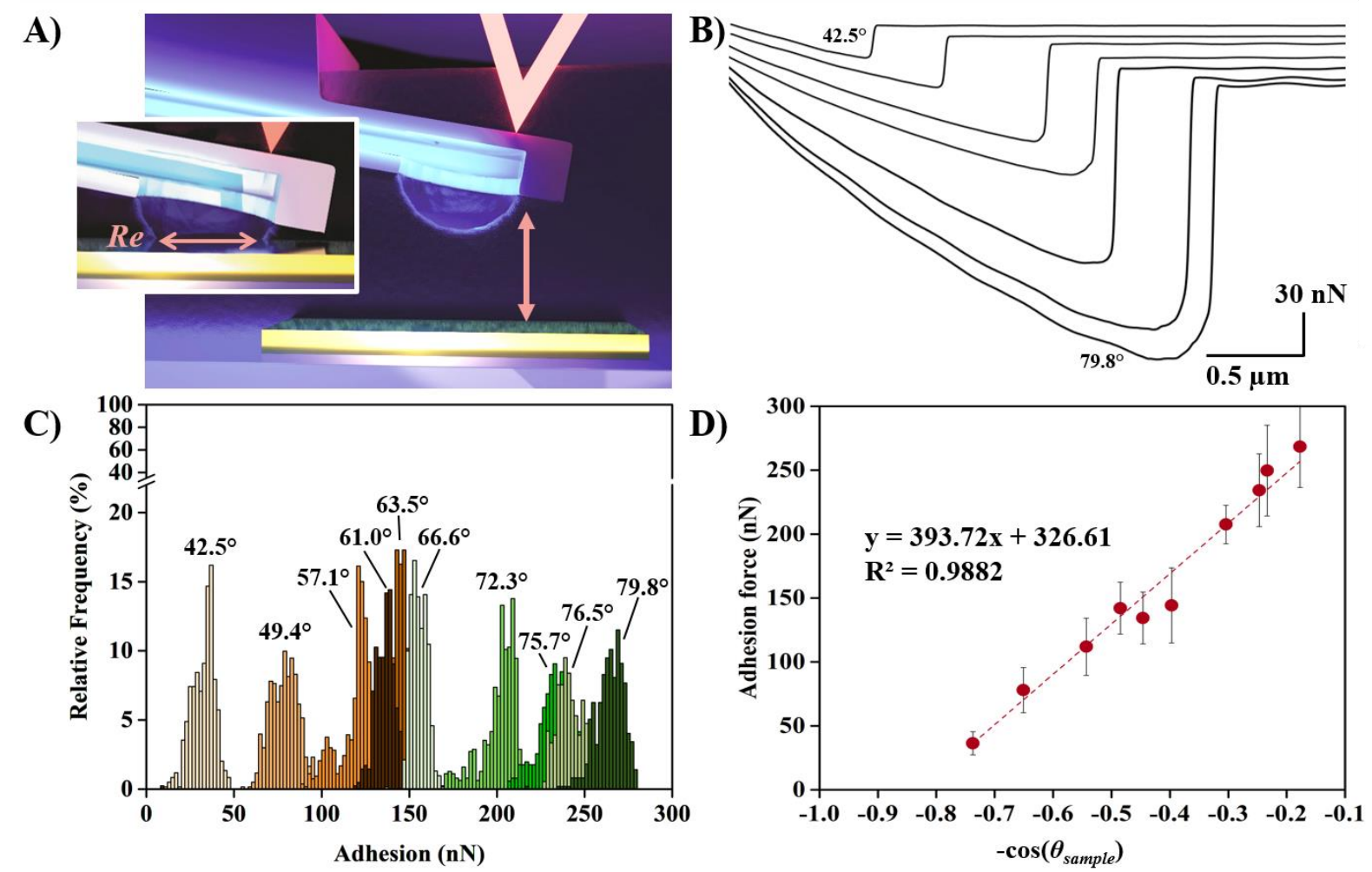

Figure 3. Probing the interactions between bubbles and hydrophobic surfaces. A) Schematic representation of the interaction between bubbles and hydrophobic surfaces. The inset shows the bubble attachment on the hydrophobic surface and what the effective bubble radius corresponds to. B) Representative force curves obtained for hydrophobic surfaces with an increasing WCA.C) Adhesion force histogram obtained for the interactions between bubbles and the different hydrophobic surfaces. For each surfaces, one representative area on the surface is presented. D) Graphic showing the variation of the adhesion force as a function of the cosine of the WCA.

Ducker and co-workers in 1994, concluded from their experiments that an air bubble in water interacts like a hydrophobic surface [19] because of the presence of long-ranged attractive forces visible on the force curves obtained between bubbles and hydrophobic surfaces, which are longer ranged than expected from the Derjaguin-Landau-Verwey-Overbeek (DLVO) theory. After that several studies followed [2,43-45], notably the study of Fielden et al., who also showed that when a bubble interacts with a hydrophobic surface, a hydrophobic force is operating in addition to DLVO forces [43]. Indeed, at close separation distances between bubbles and surfaces, forces resulting from the electrical double-layers (EDL) and van der Walls (VDW) interactions are expected to be repulsive, thus if an attractive force between bubbles and surfaces is measured, it means that hydrophobic forces are significant and that their range is longer compared to EDL forces and VDW. What happens is that when the bubble approaches a hydrophobic surface, an aqueous film will separate the two. While EDL and VDW interactions are likely to stabilize this film, the strong attraction resulting from the hydrophobic interaction will lead to the film breakage and the further bubble attachment to the surface by the formation of a three-phase contact line (TPC, three phases are surface, water, air) [46,47]. In our case, bubbles are negatively charged [48], the gold surfaces produced are neutral at the $\mathrm{pH}$ considered (7.4 [49]), thus the electrostatic interactions are not attractive. Regarding the VDW forces, although not known for our system, the Hamaker constant of the interactions air-water-hydrocarbon is negative [50]. Considering that 
hydrocarbons are hydrophobic as well as our surfaces, and because most studies involving bubble attachment to hydrophobic surfaces consider a negative Hamaker constant [51-54], then we can assume that the VDW interaction is repulsive as well. On our approach force curves, we do not observe any repulsive forces, on the contrary, as the bubble approaches the surface, we observe a "jump-in" reflecting the fact that the bubble gets suddenly attached to the surface (Supplementary Fig. S3A). This jump-in, as in the previous studies on bubble-hydrophobic surface interaction show, is most likely due to a long-range hydrophobic force that causes the disruption of the water film and the formation of the TPC line, as illustrated in Supplementary Fig. S3B.

Considering this, we thus chose to confront our data to the JKR theory [55], which allows to link the adhesion force to the work of adhesion, which is, according to the Young equation, related to the wettability of the surfaces, and thus to their WCA. More specifically, the JKR model describes the adhesion mechanism between a spherical or curved particle (the bubble in our case) and a flat surface (the hydrophobic samples in our case) in a medium (PBS in our case) [56]. It predicts that:

$$
F_{a d h}=1.5 \pi R e W_{a d h}(5)
$$

Where $F_{a d h}$ is the adhesion force recorded, $W_{a d h}$ the work of adhesion, and $R e$ is the effective radius of curvature of the bubble area in contact with the sample surface (Inset in Fig 3A). By simplifying the equation and substituting the $W_{a d h}$, the following linear equation was obtained (detailed calculations can be found in Supplementary text 1):

$$
F_{\text {adh }}=330.1-401.3 \cos \theta_{\text {sample }}(6)
$$

Thus to verify the JKR theory in our case, we plotted the adhesion force as a function of the cosine of the WCA of the samples, $-\cos \theta_{\text {sample }}$, as showed in Figure 3D. Adhesion forces increase linearly with- $\cos \theta$, and the regression equation obtained is the following:

$$
F_{\text {adh }}=326.6-393.7 \cos \theta_{\text {sample }}(7)
$$

Equation 6 agrees well with the regression equation (7) of Figure 3D. Thus our results show a good agreement between experimental and theoretical adhesion forces, proving that indeed the bubble probe behaves like a hydrophobic surface. This supports and confirms the hypothesis that the attractive forces observed between bubbles and hydrophobic surfaces are due to a hydrophobic interactions. To further argument on this point, we can compare our work to the one of Alsteens and co-authors who used in 2007 hydrophobic tips to probe the local hydrophobic forces on hydrophobic surfaces, by assembling SAMs of alkanethiols on both tips and surfaces [42]. Their results also showed a linear relation between the adhesion force and the WCA, which also led them to conclude that the forces probed were indeed hydrophobic forces. Note that the forces ranges in the Alsteens study are smaller than it is the case here, which is due to the difference in the contact area between tips (tip radius is of $\sim 20 \mathrm{~nm}$ ) and bubbles (contact area radius assumed to be of 1.2 $\mu \mathrm{m})$. Overall, from our results obtained on hydrophobic surfaces, we can conclude that the microbubble probe that we produce using FluidFM is highly sensitive to differentiate small changes in hydrophobicity, and represents a new and easy method to sensitively and accurately measure the hydrophobicity of complex systems such as cell surfaces where WCA measurements are tricky or can give misleading results.

\section{The bubble probe is a valuable tool to measure the hydrophobicity of cell surfaces}

In the next steps we assessed the interactions between bubbles and microorganisms in order to corroborate whether a bubble probe can indeed be utilized to measure and differentiate the 
hydrophobicity of living microorganisms' surfaces, which compared to the hydrophobic samples we produced, present a higher complexity. To this end, we selected three different microorganisms' species relevant for their bio-medical or industrial implications i. e., a bacterial species (Pseudomonas aeruginosa), a yeast species (Candida albicans) and a microalgae species (Chlorella vulgaris) with different cell wall compositions and thus most probably different hydrophobic properties. Cells were immobilized on glass-slides coated with ConA or PEI depending on the species and the interactions with bubbles formed by FluidFM were measured in buffer at $\mathrm{pH} 7.4$ (Figure 4A, B and C). Based on the cell's respective sizes as well as on the size ratio between cells and bubbles, the interactions were measured between bubbles and single $C$. albicans (Fig. 4E) and C. vulgaris (Fig. 4F) cells, whereas they were measured with a layer of cells in the case of $P$. aeruginosa (Fig. 4D). Indeed, while for $C$. albicans $(5-7 \mu \mathrm{m}$ in diameter) and $C$. vulgaris (3-5 $\mu \mathrm{m}$ in diameter) the cell size is in the same range as the bubble size, in the case of $P$. aeruginosa, cells are relatively small compared to the bubble (approximately $1 \mu \mathrm{m}$ in width) and the bubble could interact with the surface as well. For each species, the results were acquired on 10 different cells for each microorganism coming from at least three independent cultures. In the case of $P$. aeruginosa cell layers, the retract force curves $(n=6250$ force curves obtained from 10 cells, inset in Figure 4G) show no retract peaks, which means that bubbles do not interact at all with $P$. aeruginosa cell surface. In the case of $C$. albicans (Figure $4 \mathrm{H}$ ) a single peak occurring at the contact point is visible on the force curves with an average adhesion force of $0.4 \pm 0.2 \mathrm{nN}$ (n $=5800$ force curves obtained from 10 cells, all adhesion values can be found in Supplementary Table S2). A similar type of interaction was obtained for $C$. vulgaris-microbubble interactions (Fig. 4I); however, in this case the average adhesion force recorded was of $4.2 \pm 1.2 \mathrm{nN}(\mathrm{n}=5800$ force curves obtained from 10 cells, all adhesion values can be found in Supplementary Table S3), thus 10 times higher than for $C$. albicans. Giving the shape of the retract peaks obtained on the force curves (inset in Figure 4H and 4I) for both C. albicans and C. vulgaris, our results suggest that non-specific physico-chemical interactions are involved, most probably reflecting the hydrophobic properties of the cell surfaces. An interesting point to note is that the bubble probe is able to detect forces as low as $0.4 \mathrm{nN}$, thus showing its sensitivity. These experiments thus tend to prove that the bubble probe produced using our method is indeed a valuable tool to measure the hydrophobicity of cell surfaces. In the case of $C$. vulgaris and $C$. albicans, to confirm that the forces recorded are due to only interactions with cells, we probed the interactions between bubbles and the surfaces they are immobilized on, i.e. PEI coated glass slides for C. vulgaris and ConA coated glass slides for $C$. albicans. The results presented in Supplementary Fig. S4 show that the average adhesion force recorded between bubbles and ConA surfaces is of $212.4 \pm 21.7 \mathrm{nN}$ (Supplementary Fig. S4A, n= 2500 force curves) whereas it is of $91.7 \pm 39.7 \mathrm{nN}$ (Supplementary Fig. S4B, $n=1416$ force curves) for PEI surfaces. These adhesion forces are much higher than the ones we attribute to cells (maximum $10 \mathrm{nN}$ ), thus meaning that the bubbles produced with FluidFM have a diameter small enough to probe single cells. This is an important advantage of the FluidFM method we develop here, as understanding these interactions with single cells is key to understand the complex interplay of physico-chemical forces involved in their interactions with bubbles [57]. 

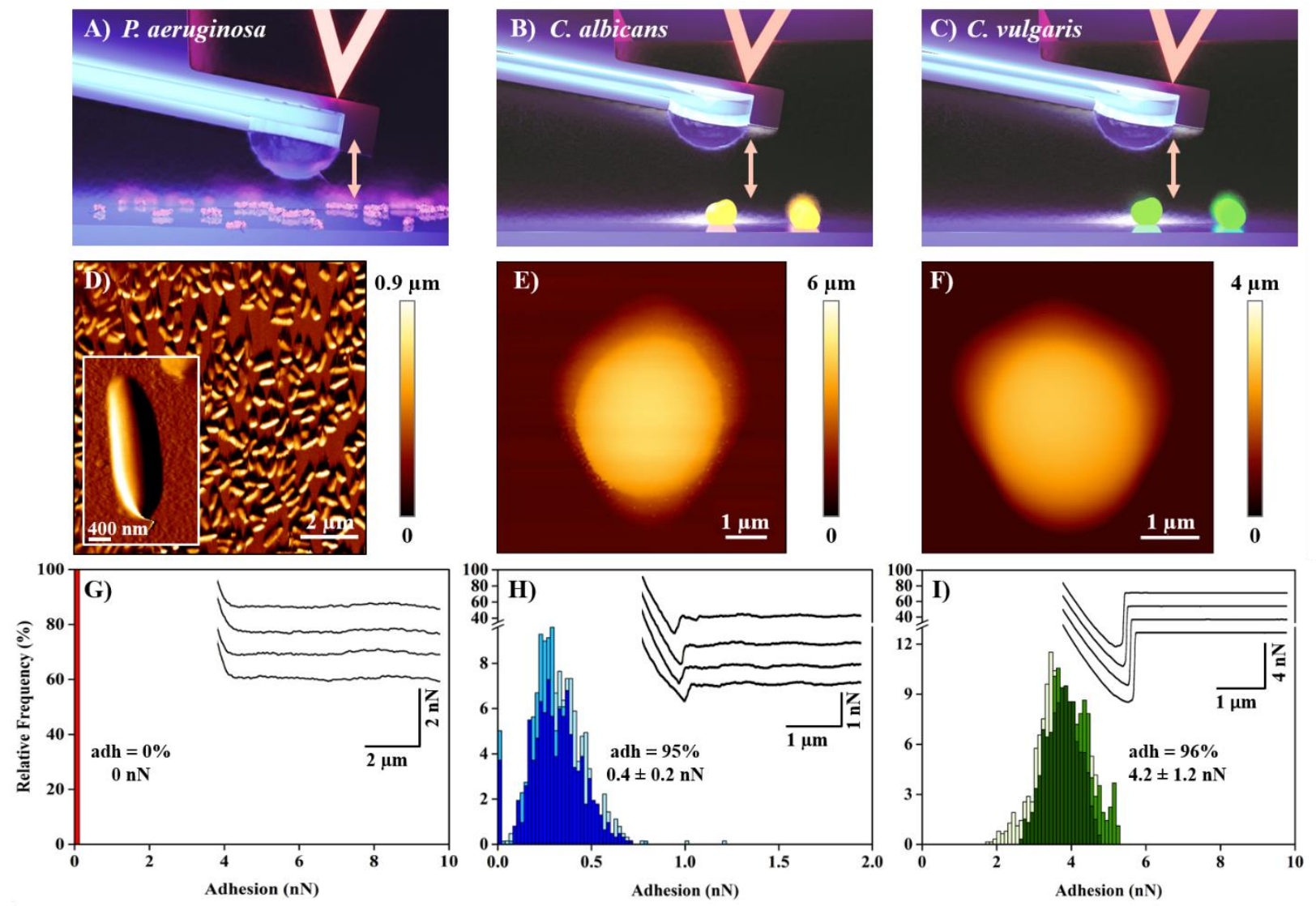

Figure 4. Probing the interactions between bubbles and microorganism. Schematic representation of A) bubble - P. aeruginosa interaction, B) bubble - C. albicans single cells interactions and C) bubble $-C$. vulgaris interactions. D) Standard AFM vertical deflection image of $P$. aeruginosa cell layers, the inset is a zoom-in of one single cell. AFM height images $E$ ) of a single $C$. albicans cell and $F$ ) of a single $C$. vulgaris cell. Adhesion force histograms obtained for $G$ ) bubble $-P$. aeruginosa interactions, $H$ ) bubble $-C$. albicans single cells interactions and I) bubble - C. vulgaris single cells interactions. In each case, 3 different cells are presented. Insets in panels $C$ ), $F$ ) and I) show representative force curves obtained in each case.

As it was previously shown in the literature, the adhesion between cells and bubbles may be hydrophobic but can further be affected by $\mathrm{pH}$, salt concentration or ionic strength [22]. To verify if this is also the case with bubbles produced by FluidFM, additional experiments were carried out with $C$. albicans to probe the interactions between single bubbles and single cells at two different $\mathrm{pH}$ (Fig. 5A-B) and at different salt concentrations (Fig. 5C). These results are presented in Figure 5. They show that when the $\mathrm{pH}$ is decreased from 7.4 to 5.2, similar nonspecific interactions are visible on the force curves but the adhesion forces recorded are higher: while they are of $0.4 \pm 0.2 \mathrm{nN}$ at $\mathrm{pH} 7.4$ (Fig. 5D), they increase to $6.2 \pm 2.5 \mathrm{nN}$ at pH 5.2 (Fig. $5 \mathrm{E}, \mathrm{n}=6249$ force curves, adhesion values obtained for 10 cells can be found in Supplementary Table S4). When we increase the salt concentration by adding $500 \mathrm{mM} \mathrm{NaCl}$ in the buffer at $\mathrm{pH}$ 5.2 , the charges present on the cells and bubbles are shielded, which results in the decrease of the adhesion forces recorded, from $6.2 \pm 2.5 \mathrm{nN}$ to $0.4 \pm 0.2 \mathrm{nN}$ in the presence of salts (Fig. $5 \mathrm{~F}, \mathrm{n}=$ 1875 force curves, adhesion values obtained for 5 cells can be found in Supplementary Table S5). These results are in line with the previous literature on the interactions of bubbles with yeast cells [22,23], as the interactions are also in our case influenced by $\mathrm{pH}$ or ionic strength. This suggests 
that in addition to hydrophobic forces, potentially other types of non-specific interactions are involved, such as electrostatic interactions.
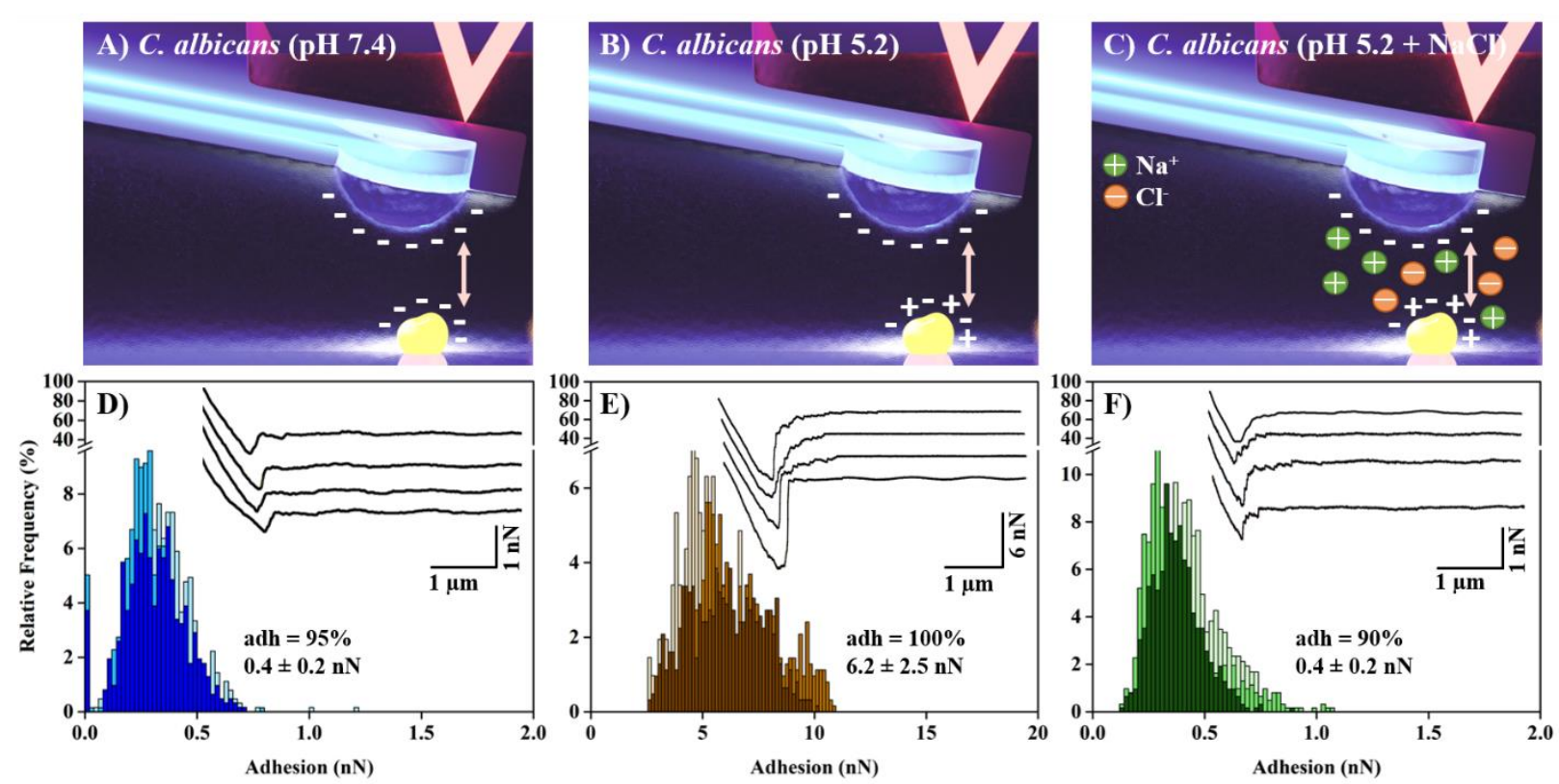

Figure 5. Characterization of the interactions between bubbles and C. albicans cells. Schematic representation of A) bubble $-C$. albicans interactions at $\mathrm{pH} 7.4, \mathrm{~B})$ bubble $-C$. albicans interactions at pH 5.2 and C) bubble - C. albicans interactions at pH 5.2 after addition of $500 \mathrm{mM}$ of $\mathrm{NaCl}$. Adhesion force histograms obtained for D) bubble $-C$. albicans interaction at $\mathrm{pH} 7.4, \mathrm{E})$ bubble $-C$. albicans interaction at $\mathrm{pH} 5.2$ and $\mathrm{F}$ ) bubble - C. albicans interaction at $\mathrm{pH} 5.2$ after addition of $500 \mathrm{mM}$ of $\mathrm{NaCl}$. In each case, 3 different cells are presented. Insets in panels D), E) and F) show representative force curves.

It is interesting to see that the interactions between bubbles and the different microorganisms result in different adhesion forces. In the case of P. aeruginosa, it is surprising that our experiments show no interactions at all with bubbles, as based on the literature, our strain of $P$. aeruginosa-(ATCC 27853) is supposed to have a hydrophobic surface [58]. To find an answer to this, we looked at the cell's surface charge, which is negative [59]. The bubbles being also negatively charged in water, it is thus possible that in this case, the electrostatic repulsion between bubbles and cells may be dominant compared to the hydrophobic force. To evaluate this, we looked at the approach curves obtained when the bubble interacts with $P$. aeruginosa cells, presented in Supplementary Fig. S5. Compared to the curves obtained on C. vulgaris or C. albicans, we observe continuously increasing repulsive forces during the approach with the bubble to the bacterial surface. These repulsive forces were recorded at a distance of more than $100 \mathrm{~nm}$, thus at longer range than the hydrophobic jump-in that we can see in the case of $C$. vulgaris, and could very well be attributed to the repulsion between the two negatively charged surfaces. What happens then is that the bubble can go to close contact with the cells but the water film between the bubble and the cell is never ruptured and the TPC line never forms. Thus in the specific case of $P$. aeruginosa, the fact that bubbles do not interact with cell surfaces seem not to reflect its hydrophobic properties, but rather its electronegativity. Regarding the interactions with $C$. albicans, the approach curves show no repulsive forces. C. albicans is known to present a cell surface hydrophobicity $[60,61]$. Thus the adhesion peaks visible on the retract force curves can be at first attributed to hydrophobic interactions between cells and bubbles. However, when the $\mathrm{pH}$ of the 
PBS buffer is decreased from 7.4 to 5.2, the adhesion forces recorded are increased by a factor of 15. This can be due to the fact that changes in the $\mathrm{pH}$ can have an impact on the tertiary structure of surface proteins by denaturation, which can lead to a change in the hydrophobicity of the cell surface [22]. However, here also the surface charge of the cells should be taken into consideration. Indeed, a study by Chen and co-workers showed that $C$. albicans cells have a more negative global charge at $\mathrm{pH} 7.4$, of $-26 \mathrm{mV}$, than at $\mathrm{pH} 5.2(-15 \mathrm{mV})$ [62]. In our case, it can thus mean that the decreased adhesion forces recorded at $\mathrm{pH} 7.4$ are due to the increased electrostatic repulsion with the negatively charged bubbles. The role of electrostatic interactions in the total force recorded is also confirmed by the experiments we performed in the presence of $500 \mathrm{mM}$ of salts, where the charges present on the surfaces are screened, which resulted in the decrease of the adhesion forces recorded. Thus in the case of $C$. albicans, compared to $P$. aeruginosa, the electrostatic repulsion does not dominate the hydrophobic force, but influences it in an important manner. Thus the forces recorded between bubbles and microorganisms result from a balance between electrostatic repulsion and hydrophobic interaction. It is then probably also the case for $C$. vulgaris, for which the approach force curves show a jump-in that can be attributed to the hydrophobic force between bubbles and cells, which is dominant compared to the electrostatic repulsion. While the cell wall composition of $C$. vulgaris is not fully known, studies have shown that $C$. vulgaris cells are hydrophobic (hydrophobic proteins available on the cell wall) and negatively charged at the $\mathrm{pH}$ considered (7.4) [63,64].

Altogether, if we compare the bubble probe to a hydrophobic AFM tip like it was developed by Alsteens et al., the bubble probe goes a step further towards the understanding of the interactions between hydrophobic interfaces and cells, by taking into account not only the hydrophobicity of the cell surfaces but also their charge. This new method will thus be of great importance to design or engineer processes in which such interactions take place. Let's give a concrete example; in cell separation processes by flotation [65-67,9], using this method will allow to predict the efficiency by determining if cells and bubbles will attach in large-scale processes.

\section{Interactions of cells with bubbles can be modulated}

In 1994 Ducker and co-workers used sodium dodecylsulfate (SDS), a surfactant that absorbs at the bubble surface to render it hydrophilic. When probing the interactions with a silica sphere, their results showed the removal of the hydrophobic force that was taking place with clean bubbles [19]. These experiments were the first to demonstrate that it is possible to modify the interaction of bubbles by modifying their surface physico-chemical properties. Thus in order to modulate the interactions between bubbles and cells, we decided to work on the possibility to functionalize the surface of bubbles produced using FluidFM with molecules expected to specifically interact with the cell wall. As a model experiment, we first used a fluorescent surfactant for direct visualization of the functionalization of the bubble interface. The strategy that we developed to functionalize bubbles consists first in filling the microchannel of the FluidFM probe with air and dipping it in a surfactant solution (Fig. 6A), followed by aspirating the amphiphilic surfactant (fluorescent surfactant or colistin) inside the front of the microchannel, by applying a negative pressure (Fig 6B). The filled cantilever is then immersed in a petri dish where the cells are immobilized in buffer. The surfactant solution is locally released by applying a positive pressure (Fig. 6C), leading to the formation of a bubble when all the surfactant solution is out (Fig. 6D). Because of the close proximity of the surfactants to the bubble, they directly assemble at bubble surface. Moreover, the silanization process we use to hydrophobize the cantilevers, under vacuum, allows also the hydrophobization of the inside of the cantilever, thus the liquid inside the cantilever can easily be ejected. The corresponding fluorescence microscopy 
images are presented in Fig. 6E-G. Fig. 6E shows a bottom-view of the cantilever filled with the fluorescent surfactant at a pressure of 0 mbar with no bubble at the aperture of the cantilever. When a pressure of 200 mbar is applied, the surfactants are ejected from the cantilever and a bubble forms at its aperture. As it can be visualized in Fig. 6F the bubble produced is fluorescent, which means that the fluorescent surfactants are present at its surface. To verify the stability of the surfactant on the gas/liquid interphase, time course images were taken using fluorescence microscopy for $15 \mathrm{~min}$. The resulting image (Fig. 6G) shows that the surfactant do not diffuse from the microbubble to its surroundings, proving that the functionalized bubble is stable over time and can be used in force spectroscopy.

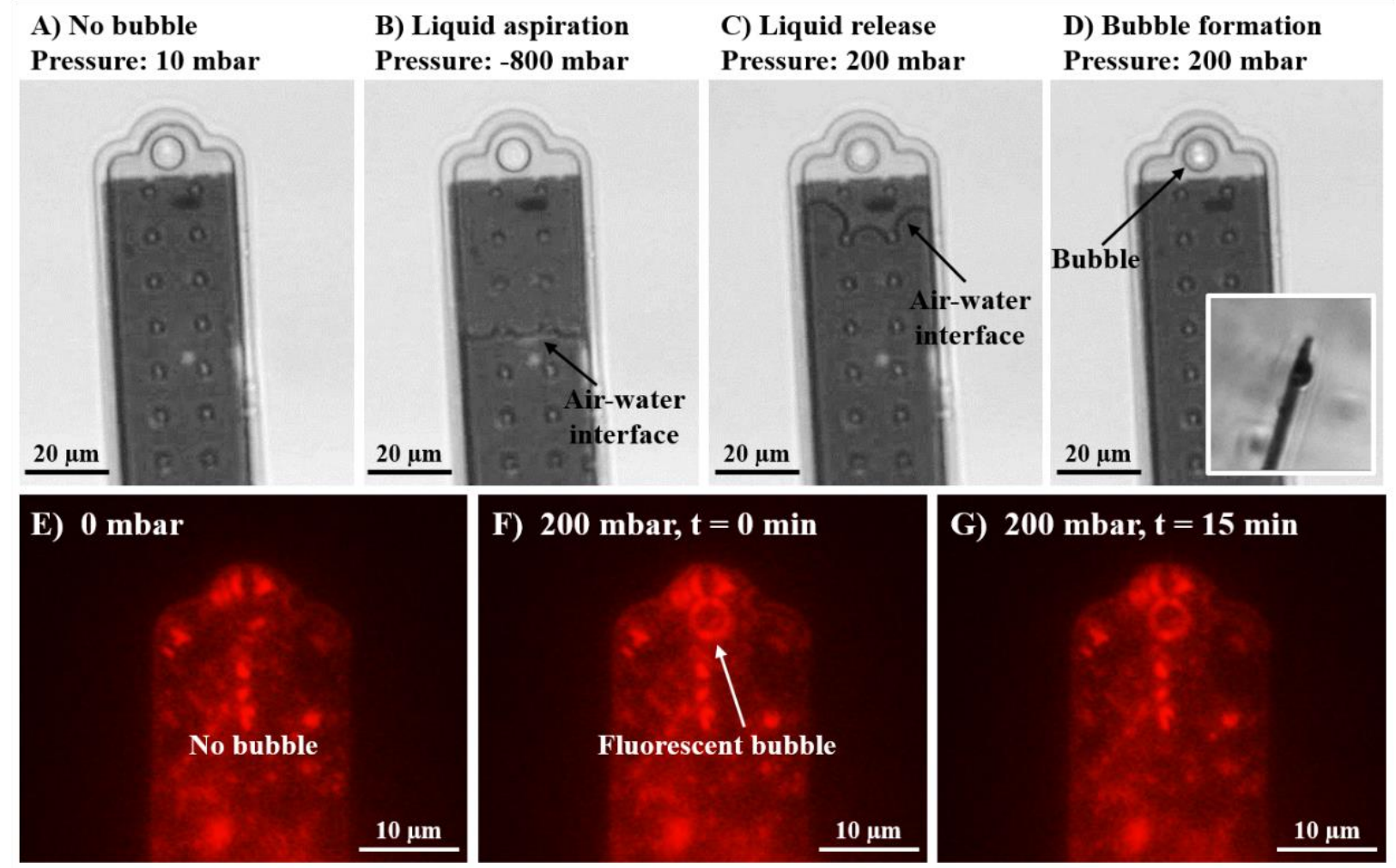

Figure 6. Functionalizing bubbles using a fluorescent surfactant. Bottom view of a FluidFM probe A) at an applied pressure of $10 \mathrm{mBar}, \mathrm{B})$ at an applied pressure of $-800 \mathrm{mBar}$ to aspirate the liquid, $C$ ) at an applied pressure of $200 \mathrm{mBar}$ to locally dispense the liquid outside of the cantilver and D) at an applied pressure of $200 \mathrm{mBar}$ pressure when a bubble forms after releasing all the liquid inside the cantilever. Fluorescence images of the bottom view of E) a FluidFM probe containing surfactants at 0 mbar, F) the same FluidFM probe at 200 mbar with a bubble formed at its aperture and D) after $15 \mathrm{~min}$.

While this model experiment demonstrates the possibility and applicability of the microbubble functionalization process, we then used this method to functionalize the bubbles with a molecule allowing to modulate its interactions with cells. As in the case of $P$. aeruginosa, bubbles do not interact with the cell wall, we thus looked for an amphiphilic molecule able to bind specifically the cell wall of this bacterial species. Colistin, also known as polymyxin E, is a natural polycyclic antibacterial peptide which specifically interacts with lipopolysaccharide (LPS) [68], the main component of $P$. aeruginosa outer cell membrane. Moreover, colistin contains both hydrophilic and hydrophobic moieties that gives it amphiphilic properties. As a first step, we probed the interactions between colistin functionalized directly on an AFM cantilever (concentration of $100 \mu \mathrm{g} / \mathrm{L}$ ) and $P$. aeruginosa cells in single-molecule force spectroscopy 
experiments (Fig. 7A). In this case the retract force curves obtained show multiple retract adhesions on rather long distances, with a maximum adhesion force of $332.1 \pm 207.2 \mathrm{pN}$ (Fig. 7D and $G n=2346$ force curves, adhesion values obtained for 10 cell areas can be found in Supplementary Table S6). These unfoldings can be attributed to the unfolding of surface polymers, notably LPS, which interacts specifically with colistin at the surface of tips. We then used colistin to functionalize the surface of bubbles (concentration of $20 \mathrm{mg} / \mathrm{L}$, Fig. 7B); the interactions obtained show a similar pattern as in the case of the functionalized AFM tips, with similar unfoldings (Fig. 7E) and a maximum average adhesion force of $590.0 \pm 317.5$ pN (Fig. 7H, $\mathrm{n}=$ 4560 force curves, adhesion values obtained for 10 cell areas can be found in Supplementary Table S7). While this force is in the same range as the one obtained with functionalized tips, it is still almost two times higher. Moreover, in this case, the unfoldings events were recorded at longer distances, up to $20 \mu \mathrm{m}$. Note that in this case, a $100 \mu \mathrm{m}$-long piezo (CellHesion module) was used to reveal the full length of these unfoldings. The fact that the unfolding pattern is similar on the force curves obtained with tips or with bubbles proves that colistin molecules are present at the surface of the bubble and interact with the bacterial surface. The difference in the adhesion force and unfolding length between the tip and the bubble is attributed to the difference in the contact area between the two systems. AFM tips have an apex curvature radius smaller than $50 \mathrm{~nm}$, thus the contact area between colistin molecules and cells is smaller compared to the bubble which has a much larger degree of curvature. Thus in the case of bubbles, more LPS molecules are involved in the interaction with more colistin, resulting in higher adhesion forces and unfolding lengths. Finally the same $100 \mu \mathrm{m}$-long piezo was used to probe the interactions between bubbles without colistin; as it was already the case in the previous experiment with a classic $15 \mu \mathrm{m}$-long piezo (Fig. 4G), no interactions with the cells were observed (Fig. 7C), as force curves shows no retract adhesion peaks (Fig. 7F and I). Moreover in this case, the repulsive electrostatic force that was visible on the force curves with clean bubbles were not visible anymore. This is due to the fact that the presence colistin on the bubble surface changes its charge. In addition, as the hydrophobic part of colistin most probably remains inside the bubble, the hydrophilic part of it is exposed on the surface interacting with the cells, thus, like in the case of SDS in Ducker's work, rendering the bubble hydrophilic. This is the reason why no hydrophobic peak is visible on the force curves in this case; the positive colistin molecules on the bubble interacts electrostatically with the LPS, which is then unfolded from the cell surface upon retraction, as proved by the long unfoldings visible on the retract force curves. These results thus demonstrate that by functionalizing the bubble produced using FluidFM, it is possible to modulate their interactions with cells. 


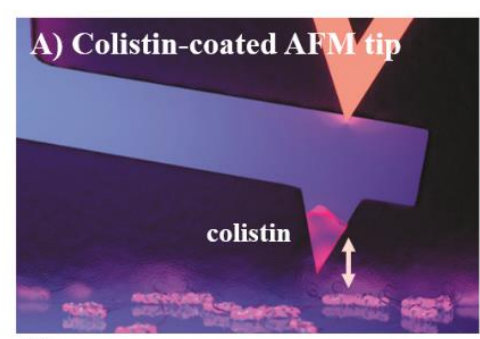

D)

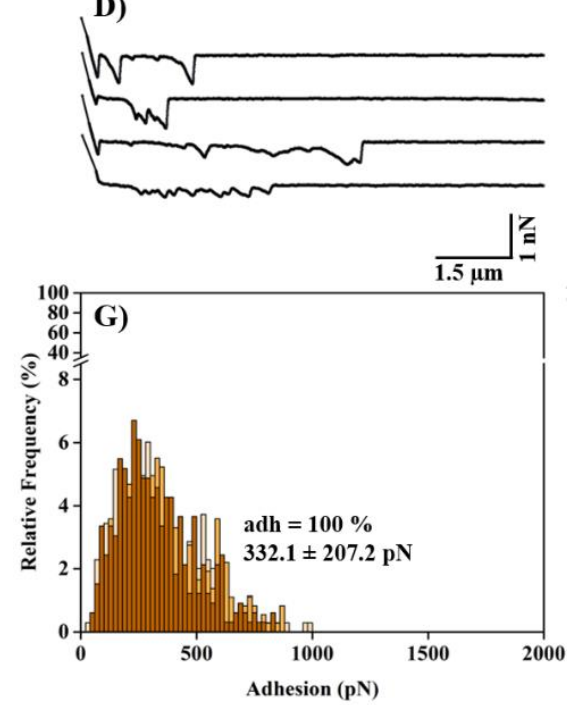

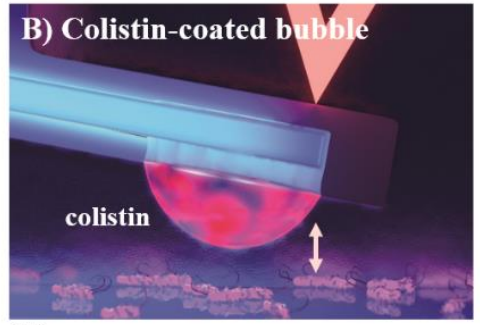

E)
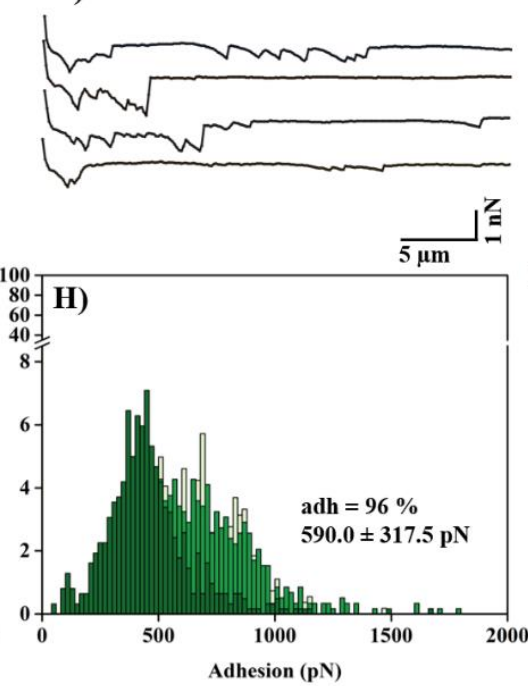

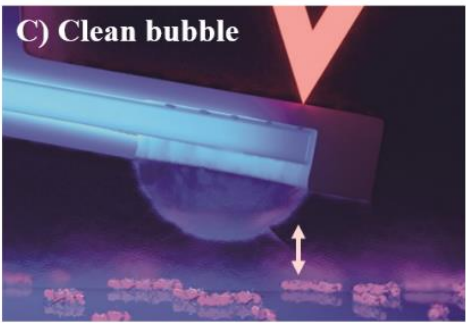

F)
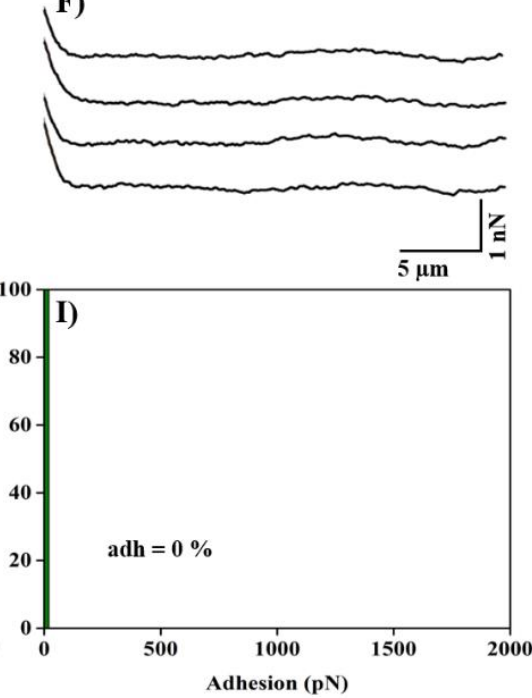

Figure 7. Modulation of the interactions between bubbles and P. aeruginosa cells by colistin. Schematic representation of A) colistin-coated AFM tips - P. aeruginosa cell layers interactions, B) colistin-coated bubbles - P. aeruginosa cell layers interactions and $C$ ) clean bubbles $-P$. aeruginosa cell layers interactions. Representative force curves obtained for D) colistin-coated AFM tips -P. aeruginosa cell layers interactions, E) colistin-coated bubbles - P. aeruginosa cell layers interactions using a 100- $\mu$ m long piezo and $F$ ) clean bubbles - P. aeruginosa cell layers interactions using a 100- $\mu$ m long piezo. Adhesion force histograms obtained for $G$ ) colistin-coated AFM tips - P. aeruginosa cell layers interactions, $H$ ) colistin-coated bubbles - P. aeruginosa cell layers interactions using a 100- $\mu \mathrm{m}$ long piezo and I) clean bubbles - P. aeruginosa cell layers interactions using a 100- $\mu \mathrm{m}$ long piezo. In each case, 3 different cells are presented

To further prove the good functionalization of bubbles with colistin in these experiments, we characterized the functionalized bubbles produced and found that at 200 mbar of pressure applied in the cantilever, their perpendicular size $\mathrm{H}$ is of $2.3 \mu \mathrm{m}$ instead of $3.2 \mu \mathrm{m}$ as it is observed from the clean bubbles (Supplementary Fig. S6). This change in the bubble size thus means that with colistin, the surface tension was modified. Using the Laplace equation, it is easy, knowing the bubble dimension, to find the surface tension, which we estimate in this case at $47 \mathrm{mN} / \mathrm{m}$. Knowing that the surface tension of PBS is of $71 \mathrm{mN} / \mathrm{m}$, this proves that the bubble surface was indeed functionalized. However when we measure the surface tension of a colistin solution at the concentration used $(20 \mathrm{mg} / \mathrm{L})$, the surface tension is unchanged compared to PBS only. This means that when using our procedure, all the colistin molecules present in the solution aspirated in the cantilever concentrate locally at the bubble surface, changing the surface tension and thus its dimensions for the same pressure applied. Note that at colistin concentrations higher than $20 \mathrm{mg} / \mathrm{L}$, no bubbles could be formed, thus probably because of the too high local concentration of colistin. What happens is that as we increase the pressure, the liquid gets ejected from the cantilever but not the colistin molecules, which stay stuck at the gas/liquid interphase. This ensures that no 
colistin molecules are free in the medium and contaminate the cell surface. Even if it was the case, given the small volume of the colistin solution aspirated (approximately up to $400 \mathrm{fL}$ ), and because this is performed far away from the surface (in the z-direction), the probability that we may measure the interaction between the bubble and colistin attached to the cell surface is very low. This final step in our study is in fact a very important step as here we provide the possibility to (i) modify easily the surface of the bubbles produced using FluidFM, and (ii) to show in what way the modification of the bubble surface influences the nature and strength of the interaction with cells. This has many implications in the different fields were bubble-microorganisms interactions take place.

\section{CONCLUSIONS}

Compared to existing AFM approaches, these new developments based on FluidFM allow to produce stable, microsized bubbles and to probe their interactions with abiotic surfaces and cell's interfaces. The fact that it is now possible to maintain the size of bubbles constant over time is the main advantage of this new method. Indeed, this ensures that the contact areas between bubbles and samples stay constant at a given applied force during force spectroscopy experiments, allowing to record adhesion forces with accuracy and reliability. While on hydrophobic samples, the results show that bubbles behave like hydrophobic surfaces, the results obtained on cells show that in addition to hydrophobic forces, interactions are also influenced by electrostatic forces. This makes this new method an ideal tool to apprehend all the complexity of bubble-microorganisms interactions. Finally, we develop a strategy to functionalize the surface of bubbles produced using FluidFM with biomolecules and we show for the first time that these modifications modify their interactions with cells. This is a major advancement as it will open the possibility to engineer bubbles and this way exploit their full potential in various application fields. For instance, our team is at the moment using this strategy to engineer a new flotation process to harvest microalgae cells, by functionalizing the bubble surface with amphiphilic chitosan, a molecule that we have already shown to promote microalgae aggregation [32]. In future projects, this strategy could also be used to specifically separate cell populations from each other; for example to separate bacterial cells from human blood cells in the case of sepsis, but many other applications can now be envisioned.

\section{ACKNOWLEDGMENT}

This work was performed thanks to a funding from Agence Nationale de la Recherche (ANR), JCJC project FLOTALG (ANR-18-CE43-000101). C.F.-D. and E.D are researchers at CNRS. The authors would like to thank Dr. Pablo Döerig for fruitful discussions on the FluidFM. This work was partly supported by LAAS-CNRS micro- and nano- technologies platform, member of the French RENATECH network. Finally we thank Yannick Carbonnaux (makitbe.com) for the artwork and illustrations.

\section{AUTHOR CONTRIBUTIONS}

Irem Demir: Methodology, Investigation, Writing — original draft, Writing — review \& editing

Ines Lüchtefeld: Methodology, Investigation, Writing — review \& editing Claude LeMen: Methodology, Investigation, Writing — review \& editing

Etienne Dague: Methodology, Conceptualization, Supervision, Writing-review \& editing Pascal Guiraud: Methodology, Conceptualization, Supervision, Writing - review \& editing Tomaso Zambelli: Methodology, Conceptualization, Supervision, Writing-review \& editing 
Cécile Formosa-Dague: Methodology, Conceptualization, Supervision, Writing — original draft, Writing_review \& editing

\section{CONFLICTS of INTEREST}

There are no conflicts to declare

\section{REFERENCES}

[1] D. Lohse, X. Zhang, Surface nanobubbles and nanodroplets, Rev. Mod. Phys. 87 (2015) 9811035. https://doi.org/10.1103/RevModPhys.87.981.

[2] J. Israelachvili, R. Pashley, The hydrophobic interaction is long range, decaying exponentially with distance, Nature. 300 (1982) 341-342. https://doi.org/10.1038/300341a0.

[3] J. Wen, Q. Sun, Z. Sun, H. Gu, An improved image processing technique for determination of volume and surface area of rising bubble, International Journal of Multiphase Flow. 104 (2018) 294-306. https://doi.org/10.1016/j.ijmultiphaseflow.2018.02.004.

[4] S.R. Sirsi, M.A. Borden, Microbubble compositions, properties and biomedical applications, Bubble Science, Engineering \& Technology. (2016). https:/www.tandfonline.com/doi/abs/10.1179/175889709X446507 (accessed February 10, 2021).

[5] J.E. George, S. Chidangil, S.D. George, Recent Progress in Fabricating Superaerophobic and Superaerophilic Surfaces, Advanced Materials Interfaces. 4 (2017) 1601088. https://doi.org/10.1002/admi.201601088.

[6] K. Minamikawa, M. Takahashi, T. Makino, K. Tago, M. Hayatsu, Irrigation with oxygennanobubble water can reduce methane emission and arsenic dissolution in a flooded rice paddy, Environ. Res. Lett. 10 (2015) 084012. https://doi.org/10.1088/17489326/10/8/084012.

[7] A. Ushida, T. Hasegawa, T. Nakajima, H. Uchiyama, T. Narumi, Drag reduction effect of nanobubble mixture flows through micro-orifices and capillaries, Experimental Thermal and Fluid Science. 39 (2012) 54-59. https://doi.org/10.1016/j.expthermflusci.2012.01.008.

[8] R. Lavrijsen, D.M.F. Hartmann, A. van den Brink, Y. Yin, B. Barcones, R.A. Duine, M.A. Verheijen, H.J.M. Swagten, B. Koopmans, Asymmetric magnetic bubble expansion under in-plane field in Pt/Co/Pt: Effect of interface engineering, Phys. Rev. B. 91 (2015) 104414. https://doi.org/10.1103/PhysRevB.91.104414.

[9] A. Besson, C. Formosa-Dague, P. Guiraud, Flocculation-flotation harvesting mechanism of Dunaliella salina: From nanoscale interpretation to industrial optimization, Water Research. 155 (2019) 352-361. https://doi.org/10.1016/j.watres.2019.02.043.

[10] T. Ndikubwimana, J. Chang, Z. Xiao, W. Shao, X. Zeng, I.-S. Ng, Y. Lu, Flotation: A promising microalgae harvesting and dewatering technology for biofuels production, Biotechnology and Applied Biochemistry. (2016) 315-326. https://doi.org/10.1002/biot.201500175@10.1002/(ISSN)14708744(CAT)VirtualIssues(VI)BiotechnologyinChinacollection.

[11] Z. Wu, H. Chen, Y. Dong, H. Mao, J. Sun, S. Chen, V.S.J. Craig, J. Hu, Cleaning using nanobubbles: Defouling by electrochemical generation of bubbles, Journal of Colloid and Interface Science. 328 (2008) 10-14. https://doi.org/10.1016/j.jcis.2008.08.064.

[12] A. Delalande, M. Postema, N. Mignet, P. Midoux, C. Pichon, Ultrasound and microbubbleassisted gene delivery: recent advances and ongoing challenges, Therapeutic Delivery. 3 (2012) 1199-1215. https://doi.org/10.4155/tde.12.100. 
[13] Z. Gao, A.M. Kennedy, D.A. Christensen, N.Y. Rapoport, Drug-loaded nano/microbubbles for combining ultrasonography and targeted chemotherapy, Ultrasonics. 48 (2008) 260-270. https://doi.org/10.1016/j.ultras.2007.11.002.

[14] E. Unger, T. Porter, J. Lindner, P. Grayburn, Cardiovascular drug delivery with ultrasound and microbubbles, Advanced Drug Delivery Reviews. 72 (2014) 110-126. https://doi.org/10.1016/j.addr.2014.01.012.

[15] S. Poulain, L. Bourouiba, Biosurfactants Change the Thinning of Contaminated Bubbles at Bacteria-Laden Water Interfaces, Phys. Rev. Lett. $121 \quad$ (2018) 204502. https://doi.org/10.1103/PhysRevLett.121.204502.

[16] P.L.L. Walls, J.C. Bird, L. Bourouiba, Moving with Bubbles: A Review of the Interactions between Bubbles and the Microorganisms that Surround them, Integr Comp Biol. 54 (2014) 1014-1025. https://doi.org/10.1093/icb/icu100.

[17] Y. Xing, X. Gui, L. Pan, B.-E. Pinchasik, Y. Cao, J. Liu, M. Kappl, H.-J. Butt, Recent experimental advances for understanding bubble-particle attachment in flotation, Advances in Colloid and Interface Science. 246 (2017) 105-132. https://doi.org/10.1016/j.cis.2017.05.019.

[18] G. Binnig, C.F. Quate, Ch. Gerber, Atomic Force Microscope, Phys. Rev. Lett. 56 (1986) 930-933. https://doi.org/10.1103/PhysRevLett.56.930.

[19] W.A. Ducker, Z. Xu, J.N. Israelachvili, Measurements of Hydrophobic and DLVO Forces in Bubble-Surface Interactions in Aqueous Solutions, Langmuir. 10 (1994) 3279-3289. https://doi.org/10.1021/la00021a061.

[20] W.A. Ducker, T.J. Senden, R.M. Pashley, Direct measurement of colloidal forces using an atomic force microscope, Nature. 353 (1991) 239-241. https://doi.org/10.1038/353239a0.

[21] I.U. Vakarelski, J. Lee, R.R. Dagastine, D.Y.C. Chan, G.W. Stevens, F. Grieser, Bubble Colloidal AFM Probes Formed from Ultrasonically Generated Bubbles, Langmuir. 24 (2008) 603-605. https://doi.org/10.1021/la7032059.

[22] L. Ditscherlein, S. Jolan Gulden, S. Müller, R.-P. Baumann, U.A. Peuker, H. Nirschl, Measuring interactions between yeast cells and a micro-sized air bubble via atomic force microscopy, Journal of Colloid and Interface Science. 532 (2018) 689-699. https://doi.org/10.1016/j.jcis.2018.08.031.

[23] S. Yumiyama, S. Kato, Y. Konishi, T. Nomura, Direct measurement of interaction forces between a yeast cell and a microbubble using atomic force microscopy, Colloids and Surfaces A: Physicochemical and Engineering Aspects. (2019) 123963. https://doi.org/10.1016/j.colsurfa.2019.123963.

[24] J.C. Eriksson, S. Ljunggren, On the mechanically unstable free energy minimum of a gas bubble which is submerged in water and adheres to a hydrophobic wall, Colloids and Surfaces A: Physicochemical and Engineering Aspects. 159 (1999) 159-163. https://doi.org/10.1016/S0927-7757(99)00171-5.

[25] S. Ljunggren, J.C. Eriksson, The lifetime of a colloid-sized gas bubble in water and the cause of the hydrophobic attraction, Colloids and Surfaces A: Physicochemical and Engineering Aspects. 129-130 (1997) 151-155. https://doi.org/10.1016/S0927-7757(97)00033-2.

[26] H.-J. Butt, M. Farshchi-Tabrizi, M. Kappl, Using capillary forces to determine the geometry of nanocontacts, Journal of Applied Physics. $100 \quad$ (2006) 024312. https://doi.org/10.1063/1.2210188. 
[27] G. Gillies, M. Kappl, H.-J. Butt, Direct measurements of particle-bubble interactions, Advances in Colloid and Interface Science. 114-115 (2005) 165-172. https://doi.org/10.1016/j.cis.2004.08.003.

[28] D.J. Johnson, N.J. Miles, N. Hilal, Quantification of particle-bubble interactions using atomic force microscopy: A review, Advances in Colloid and Interface Science. 127 (2006) 67-81. https://doi.org/10.1016/j.cis.2006.11.005.

[29] A. Meister, M. Gabi, P. Behr, P. Studer, J. Vörös, P. Niedermann, J. Bitterli, J. Polesel-Maris, M. Liley, H. Heinzelmann, T. Zambelli, FluidFM: Combining Atomic Force Microscopy and Nanofluidics in a Universal Liquid Delivery System for Single Cell Applications and Beyond, Nano Lett. 9 (2009) 2501-2507. https://doi.org/10.1021/n1901384x.

[30] L. Hirt, R.R. Grüter, T. Berthelot, R. Cornut, J. Vörös, T. Zambelli, Local surface modification via confined electrochemical deposition with FluidFM, RSC Adv. 5 (2015) 84517-84522. https://doi.org/10.1039/C5RA07239E.

[31] E. Potthoff, D. Ossola, T. Zambelli, J.A. Vorholt, Bacterial adhesion force quantification by fluidic force microscopy, Nanoscale. 7 (2015) 4070-4079. https://doi.org/10.1039/C4NR06495J.

[32] I. Demir, J. Blockx, E. Dague, P. Guiraud, W. Thielemans, K. Muylaert, C. Formosa-Dague, Nanoscale Evidence Unravels Microalgae Flocculation Mechanism Induced by Chitosan, ACS Appl. Bio Mater. 3 (2020) 8446-8459. https://doi.org/10.1021/acsabm.0c00772.

[33] R.R.L. Guillard, C.J. Lorenzen, Yellow-green algae with chlorophyllide C2, Journal of Phycology. 8 (1972) 10-14.

[34] G. Francius, B. Tesson, E. Dague, V. Martin-Jézéquel, Y.F. Dufrêne, Nanostructure and nanomechanics of live Phaeodactylum tricornutum morphotypes, Environ. Microbiol. 10 (2008) 1344-1356. https://doi.org/10.1111/j.1462-2920.2007.01551.x.

[35] E. Dague, D. Alsteens, J.-P. Latgé, C. Verbelen, D. Raze, A.R. Baulard, Y.F. Dufrêne, Chemical Force Microscopy of Single Live Cells, Nano Lett. 7 (2007) 3026-3030. https://doi.org/10.1021/n1071476k.

[36] R. Courson, M. Fouet, P. Joseph, F. Mesnilgrente, V. Conédéra, A.M. Gué, SAMs VAPOR DEPOSITION: A READY TO USE FUNCTIONALIZATION TECHNOLOGY FOR MONITORING WETTABILITY PROPERTIES IN MICROFLUIDIC DEVICES, 2014. https://hal.archives-ouvertes.fr/hal-01082680 (accessed January 21, 2021).

[37] J.L. Hutter, J. Bechhoefer, Calibration of atomic-force microscope tips, Review of Scientific Instruments. 64 (1993) 1868-1873. https://doi.org/10.1063/1.1143970.

[38] E. Jauvert, E. Dague, M. Séverac, L. Ressier, A.-M. Caminade, J.-P. Majoral, E. Trévisiol, Probing single molecule interactions by AFM using bio-functionalized dendritips, Sensors and Actuators B: Chemical. 168 (2012) 436-441. https://doi.org/10.1016/j.snb.2012.04.048.

[39] K. Beicker, E.T. O’Brien, M.R. Falvo, R. Superfine, Vertical Light Sheet Enhanced SideView Imaging for AFM Cell Mechanics Studies, Scientific Reports. 8 (2018) 1504. https://doi.org/10.1038/s41598-018-19791-3.

[40] E. Nelsen, C.M. Hobson, M.E. Kern, J.P. Hsiao, E.T. O’Brien Iii, T. Watanabe, B.M. Condon, M. Boyce, S. Grinstein, K.M. Hahn, M.R. Falvo, R. Superfine, Combined Atomic Force Microscope and Volumetric Light Sheet System for Correlative Force and Fluorescence Mechanobiology Studies, Scientific Reports. $10 \quad$ (2020) 8133. https://doi.org/10.1038/s41598-020-65205-8. 
[41] R.F. Tabor, F. Grieser, R.R. Dagastine, D.Y.C. Chan, Measurement and analysis of forces in bubble and droplet systems using AFM, Journal of Colloid and Interface Science. 371 (2012) 1-14. https://doi.org/10.1016/j.jcis.2011.12.047.

[42] D. Alsteens, E. Dague, P.G. Rouxhet, A.R. Baulard, Y.F. Dufrêne, Direct Measurement of Hydrophobic Forces on Cell Surfaces Using AFM, Langmuir. 23 (2007) 11977-11979. https://doi.org/10.1021/la702765c.

[43] M.L. Fielden, R.A. Hayes, J. Ralston, Surface and Capillary Forces Affecting Air Bubble-Particle Interactions in Aqueous Electrolyte, Langmuir. 12 (1996) 3721-3727. https://doi.org/10.1021/la960145c.

[44] V.S.J. Craig, B.W. Ninham, R.M. Pashley, Direct Measurement of Hydrophobic Forces: A Study of Dissolved Gas, Approach Rate, and Neutron Irradiation, Langmuir. 15 (1999) 15621569. https://doi.org/10.1021/la9805793.

[45] J.N. Israelachvili, Intermolecular and Surface Forces, Academic Press, 2011.

[46] C. Shi, X. Cui, L. Xie, Q. Liu, D.Y.C. Chan, J.N. Israelachvili, H. Zeng, Measuring Forces and Spatiotemporal Evolution of Thin Water Films between an Air Bubble and Solid Surfaces of Different Hydrophobicity, ACS Nano. 9 (2015) 95-104. https://doi.org/10.1021/nn506601j.

[47] X. Cui, C. Shi, L. Xie, J. Liu, H. Zeng, Probing Interactions between Air Bubble and Hydrophobic Polymer Surface: Impact of Solution Salinity and Interfacial Nanobubbles, Langmuir. 32 (2016) 11236-11244. https://doi.org/10.1021/acs.langmuir.6b01674.

[48] C. Yang, T. Dabros, D. Li, J. Czarnecki, J.H. Masliyah, Measurement of the Zeta Potential of Gas Bubbles in Aqueous Solutions by Microelectrophoresis Method, Journal of Colloid and Interface Science. 243 (2001) 128-135. https://doi.org/10.1006/jcis.2001.7842.

[49] Y. Zheng, W. Zheng, D. Zhu, H. Chang, Theoretical modeling of pKa's of thiol compounds in aqueous solution, New J. Chem. 43 (2019) 5239-5254. https://doi.org/10.1039/C8NJ06259E.

[50] D.B. Hough, L.R. White, The calculation of hamaker constants from liftshitz theory with applications to wetting phenomena, Advances in Colloid and Interface Science. 14 (1980) 341. https://doi.org/10.1016/0001-8686(80)80006-6.

[51] A. Gomez-Flores, S.A. Bradford, G. Hwang, G.W. Heyes, H. Kim, Particle-bubble interaction energies for particles with physical and chemical heterogeneities, Minerals Engineering. 155 (2020) 106472. https://doi.org/10.1016/j.mineng.2020.106472.

[52] N. Ishida, Direct measurement of hydrophobic particle-bubble interactions in aqueous solutions by atomic force microscopy: Effect of particle hydrophobicity, Colloids and Surfaces A: Physicochemical and Engineering Aspects. 300 (2007) 293-299. https://doi.org/10.1016/j.colsurfa.2007.02.003.

[53] J. Ralston, D. Fornasiero, R. Hayes, Bubble-particle attachment and detachment in flotation, International Journal of Mineral Processing. $56 \quad$ (1999) 133-164. https://doi.org/10.1016/S0301-7516(98)00046-5.

[54] S. Usui, E. Barouch, Effect of adsorbed layers on the van der Waals interaction between particles and bubbles in aqueous media, Journal of Colloid and Interface Science. 137 (1990) 281-288. https://doi.org/10.1016/0021-9797(90)90062-S.

[55] K.L. Johnson, K. Kendall, A.D. Roberts, D. Tabor, Surface energy and the contact of elastic solids, Proceedings of the Royal Society of London. A. Mathematical and Physical Sciences. 324 (1971) 301-313. https://doi.org/10.1098/rspa.1971.0141. 
[56] E.W. van der Vegte, G. Hadziioannou, Scanning Force Microscopy with Chemical Specificity: An Extensive Study of Chemically Specific Tip-Surface Interactions and the Chemical Imaging of Surface Functional Groups, Langmuir. 13 (1997) 4357-4368. https://doi.org/10.1021/la970025k.

[57] H.J. Busscher, W. Norde, H.C. van der Mei, Specific Molecular Recognition and Nonspecific Contributions to Bacterial Interaction Forces, Appl. Environ. Microbiol. 74 (2008) 25592564. https://doi.org/10.1128/AEM.02839-07.

[58] Y. Zhang, R.M. Miller, Effect of a Pseudomonas rhamnolipid biosurfactant on cell hydrophobicity and biodegradation of octadecane., Appl. Environ. Microbiol. 60 (1994) 2101-2106.

[59] S.A. Makin, T.J. Beveridge, The influence of A-band and B-band lipopolysaccharide on the surface characteristics and adhesion of Pseudomonas aeruginosa to surfaces, Microbiology,. 142 (1996) 299-307. https://doi.org/10.1099/13500872-142-2-299.

[60] R.J. Doyle, M. Rosenberg, Microbial cell surface hydrophobicity, American Society for Microbiology, 1990.

[61] K. HAZEN, Cell surface hydrophobicity of medically impotant fungi, especially Candida species, Microbial Cell Sueface Hydrophobicity. (1990) 249-295.

[62] H. Chen, Y. Zhou, X. Zhou, B. Liao, H.H.K. Xu, C.-H. Chu, L. Cheng, B. Ren, Dimethylaminododecyl methacrylate inhibits Candida albicans and oropharyngeal candidiasis in a pH-dependent manner, Appl Microbiol Biotechnol. 104 (2020) 3585-3595. https://doi.org/10.1007/s00253-020-10496-0.

[63] S. Hadjoudja, V. Deluchat, M. Baudu, Cell surface characterisation of Microcystis aeruginosa and Chlorella vulgaris, Journal of Colloid and Interface Science. 342 (2010) 293-299. https://doi.org/10.1016/j.jcis.2009.10.078.

[64] Y.-L. Cheng, Y.-C. Juang, G.-Y. Liao, S.-H. Ho, K.-L. Yeh, C.-Y. Chen, J.-S. Chang, J.-C. Liu, D.-J. Lee, Dispersed ozone flotation of Chlorella vulgaris, Bioresource Technology. 101 (2010) 9092-9096. https://doi.org/10.1016/j.biortech.2010.07.016.

[65] A. Besson, P. Guiraud, High-pH-induced flocculation-flotation of the hypersaline microalga Dunaliella salina, Bioresource Technology. $147 \quad$ (2013) 464-470. https://doi.org/10.1016/j.biortech.2013.08.053.

[66] C. Formosa-Dague, V. Gernigon, M. Castelain, F. Daboussi, P. Guiraud, Towards a better understanding of the flocculation/flotation mechanism of the marine microalgae Phaeodactylum tricornutum under increased $\mathrm{pH}$ using atomic force microscopy, Algal Research. 33 (2018) 369-378. https://doi.org/10.1016/j.algal.2018.06.010.

[67] J.B. Vergnes, V. Gernigon, P. Guiraud, C. Formosa-Dague, Bicarbonate Concentration Induces Production of Exopolysaccharides by Arthrospira platensis That Mediate Bioflocculation and Enhance Flotation Harvesting Efficiency, ACS Sustainable Chem. Eng. 7 (2019) 13796-13804. https://doi.org/10.1021/acssuschemeng.9b01591.

[68] D. Yahav, L. Farbman, L. Leibovici, M. Paul, Colistin: new lessons on an old antibiotic, Clin Microbiol Infect. 18 (2012) 18-29. https://doi.org/10.1111/j.1469-0691.2011.03734.x. 